\title{
molecules
}

ISSN 1420-3049

www.mdpi.com/journal/molecules

Article

\section{Synthesis and Biological Evaluation of New 2-Azetidinones with Sulfonamide Structures}

Oana Maria Dragostin ${ }^{1}$, Florentina Lupascu ${ }^{1}$, Cornelia Vasile ${ }^{2}$, Mihai Mares ${ }^{3}$, Valentin Nastasa ${ }^{3}$, Ramona Florina Moraru ${ }^{3}$, Dragos Pieptu ${ }^{4, *}$ and Lenuta Profire ${ }^{1, *}$

1 Department of Pharmaceutical Chemistry, Faculty of Pharmacy, Grigore T. Popa University of Medicine and Pharmacy, 16 University Street, Iasi 700115, Romania;

E-Mails: farmacist_oanamaria@yahoo.com (O.M.D.); geani0407@yahoo.com (F.L.)

2 Department of Physical Chemistry of Polymers, Petru Poni Institute of Macromolecular Chemistry, Romanian Academy, 41A Grigore Ghica Voda Alley, Iasi 700487, Romania;

E-Mail: cvasile@icmpp.ro

3 Laboratory of Antimicrobial Chemotherapy, Faculty of Veterinary Medicine, Ion Ionescu de la Brad University of Agricultural Sciences and Veterinary Medicine, 8 Mihail Sadoveanu Alley, Iasi 700489, Romania; E-Mails: mycomedica@gmail.com (M.M.); alvali2003@yahoo.com (V.N.); moraru82@gmail.com (R.F.M.)

4 Department of Plastic Surgery, Faculty of Medicine, Grigore T. Popa University of Medicine and Pharmacy, 16 University Street, Iasi 700115, Romania

* Authors to whom correspondence should be addressed; E-Mails: dpieptu@chpla.umfiasi.ro (D.P.); nprofire@yahoo.com (L.P.); Tel.: +40-232-412375 (L.P.); Fax: +40-232-211818 (L.P.).

Received: 26 February 2013; in revised form: 26 March 2013 / Accepted: 3 April 2013 /

Published: 8 April 2013

Abstract: New series of $N$-(arylidene)hydrazinoacetyl sulfonamides $\mathbf{4} \mathbf{a}_{1-6}, \mathbf{4} \mathbf{b}_{1-6}$ and $N$-(4-aryl-3-chloro-2-oxoazetidin-1-yl)aminoacetyl sulfonamides $\mathbf{5} \mathbf{a}_{1-6}, \mathbf{5} \mathbf{b}_{1-6}$ were synthesized. The structures of the new derivatives was confirmed using spectral methods (FT-IR, $\left.{ }^{1} \mathrm{H}-\mathrm{NMR},{ }^{13} \mathrm{C}-\mathrm{NMR}\right)$. The antibacterial activities of these compounds against Gram positive (Staphyloccoccus aureus ATCC 6583, Staphyloccoccus epidermidis ATCC 12228, Enterococcus faecalis ATCC 25912) and Gram negative (Klebsiella pneumoniae CIP 53153, Proteus vulgaris CIP 104989, Citrobacter freundii CIP 5732, Enterobacter cloacae CIP 103475, Escherichia coli ATCC 25922, Pseudomonas aeruginosa CIP 82118) bacterial strains were evaluated using the broth micro-dilution method. Compound $\mathbf{4} \mathbf{a}_{2}$ displayed the highest antibacterial activity, especially against Staphyloccoccus epidermidis, Enterococcus faecalis and Pseudomonas aeruginosa. The antioxidant potential of the 
synthesized compounds was also investigated according to ferric reducing power, total antioxidant activity and DPPH radical scavenging assays. All tested compounds showed excellent antioxidant activity in comparison with sulfadiazine and sulfisoxazole which were used as parent sulfonamides. Moreover, some of them showed an antioxidant activity comparable with that of ascorbic acid. In general, the compounds designed based on a sulfadiazine skeleton (compounds $\mathbf{4 \mathbf { a } _ { 1 - 6 }}, \mathbf{5 \mathbf { a } _ { 1 - 6 }}$ ) are more active than those obtained from sulfisoxazole (compounds $\mathbf{4} \mathbf{b}_{1-6}, \mathbf{5 b}_{1-6}$ ), and the $N$-(arylidene)hydrazinoacetyl sulfonamide derivatives $\mathbf{4} \mathbf{a}_{1-\mathbf{6}}, \mathbf{4} \mathbf{b}_{1-\mathbf{6}}$ are more active than their azetidionone analogues $\mathbf{5} \mathbf{a}_{1-6}, \mathbf{5} \mathbf{b}_{1-6}$.

Keywords: sulfonamide; azetidinone; synthesis; antimicrobial activity; antioxidant effect

\section{Introduction}

The 2-azetidinone skeleton, otherwise known as the $\beta$-lactam ring, has been recognized as a useful building block in the synthesis of biologically important compounds. Azetindin-2-one derivatives display interesting biological activities such as antifungal, antimicrobial [1-4], antitubercular [5,6], analgesic, anti-inflammatory [7,8], chymase inhibitory [9], antitumoral [10-12], antiviral, antidiabetic and cholesterol absorption inhibitory properties [13]. The activity of famous antibiotic classes such as the penicillins, cephalosporins, carumonam, aztreonam, thienamicine, nocardicins and carbapenems is attributed to the presence of an 2-azetidinone ring [2]. Unfortunately, the most widely used of them exert selective pressure on bacteria and permit the proliferation of resistant organisms. Several synthetic and semi-synthetic $\beta$-lactam antibiotics were developed due to the growing resistance of bacteria towards the classical $\beta$-lactam antibiotics and the need for drugs with a more specific antibacterial activity [1]. The biological activity of sulfonamides is also well documented. They have be found to be useful in a variety of applications, including antibacterial, antifungal, antitumor agents, diuretics, carbonic anhydrase inhibitors, hypoglycemic agents, thyroid inhibitors, anticonvulsants and protease inhibitors $[14,15]$. Among antibacterial sulfonamides, sulfadiazine and its silver and cerium salts have an important place. They are widely used as topical agents for the management of burns where they prevent infections and promote rapid healing with minimal scarring [15].

Wounds are physical injuries that result in an opening of the skin. Proper healing of wounds is essential for the restoration of disrupted anatomical continuity and disturbed functional status of the skin [16]. Normal healing of wounds is a dynamic process following three phases: inflammation, granulation (tissue formation) and re-epithelization (tissue remodeling), which overlap in time [17]. It was proven that reactive oxygen species (ROS) and bacterial infections are deleterious to the wound healing process due to their harmful effects on cells and tissues [18]. ROS are produced in high amounts at wound sites as a defense mechanism against invading bacteria. At the same time, the process of wound healing may be hampered by the presence of free radicals, which can damage the cells surrounding the wound, or by microbial infection [19] and recent data has proved the beneficial effects of antioxidants in the wound healing process [20-22]. In the present study, we are reporting the design, synthesis and biological evaluation of some new 2-azetidinone derivatives of sulfadiazine and sulfisoxazole with potential use in wound healing processes. 


\section{Results and Discussion}

\subsection{Chemistry}

Azetidinone derivatives $\mathbf{5} \mathbf{a}_{1-6}, \mathbf{5 b}_{1-6}$ were prepared using the method summarized in Scheme 1. First, sulfadiazine (4-amino- $N$-pyrimidin-2-yl-benzensulfonamide, 1a) and sulfisoxazole [4-amino- $N$ (3,4-dimethyl-1,2-oxazol-5-yl)benzensulfonamide, 1b] were reacted with chloroacetyl chloride whereby the corresponding chloracetyl derivatives $\mathbf{2 a}-\mathbf{b}$ were obtained. Compounds $\mathbf{2 a}-\mathbf{b}$ on amination with hydrazine hydrate afforded hydrazinoacetyl sulfonamide derivatives $\mathbf{3 a}-\mathbf{b}$ [23]. The condensation reaction of compounds $\mathbf{3 a}-\mathbf{b}$ with various aromatic aldehydes yielded $N$-(arylidene)hydrazinoacetyl sulfonamide derivatives $\mathbf{4} \mathbf{a}_{1-6}, \mathbf{4} \mathbf{b}_{1-6}$. Finally, the compounds $4 \mathbf{a}_{1-6}, \mathbf{4 b}_{1-6}$ upon reaction with chloracetyl chloride in the presence of triethylamine afforded $N$-(4-aryl-3-chloro-2-oxoazetidin-1yl)aminoacetyl sulfonamides $\mathbf{5} \mathbf{a}_{1-6}, \mathbf{5} \mathbf{b}_{1-6}$.

Scheme 1. Synthesis of azetidinone derivatives $\left(\mathbf{5} \mathbf{a}_{1-6}, \mathbf{5} \mathbf{b}_{1-6}\right)$.

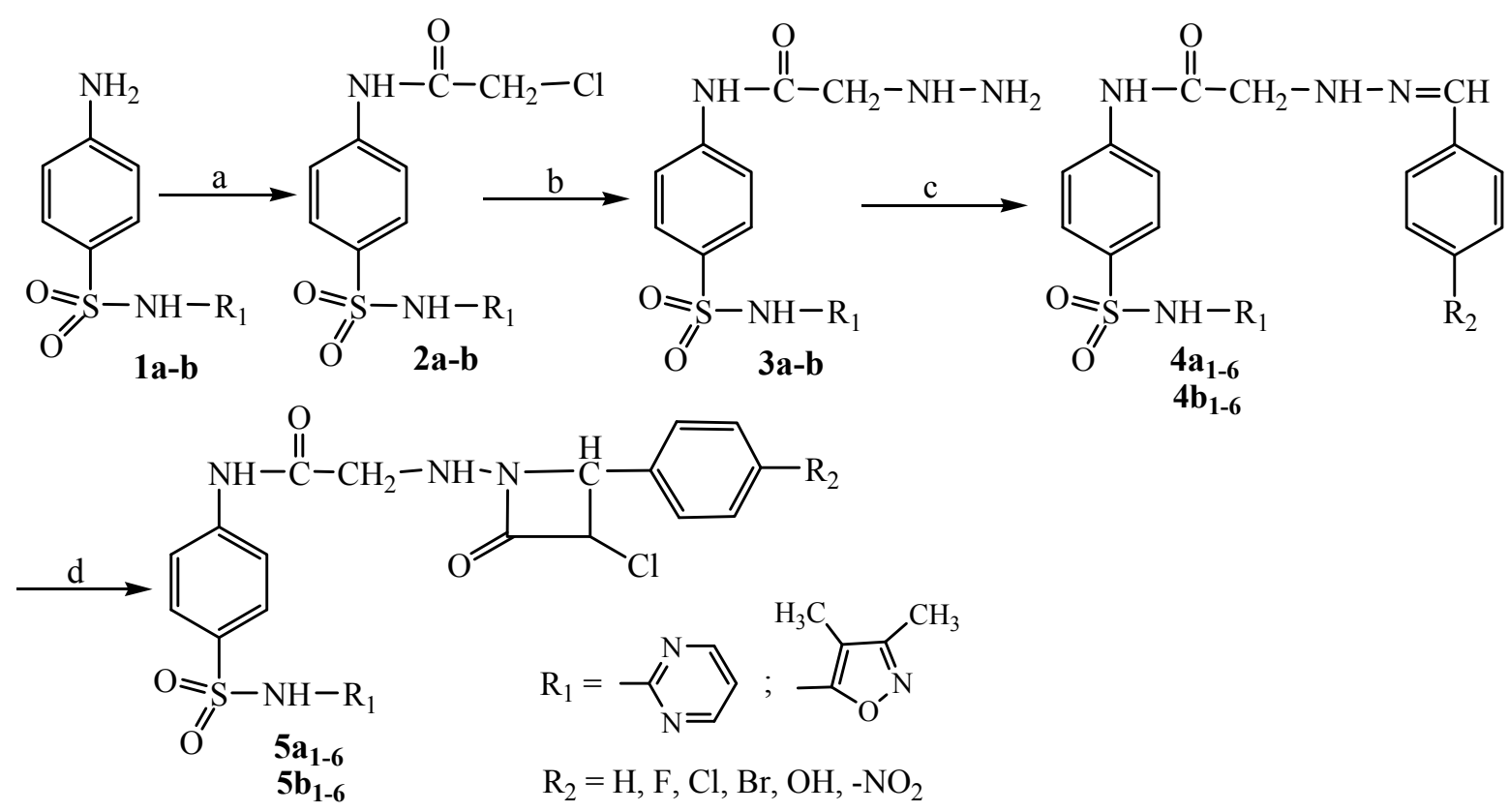

Reagents and Conditions: (a) chloracetyl chloride, dry acetone, anhydrous $\mathrm{K}_{2} \mathrm{CO}_{3}$, heating $12 \mathrm{~h}$; (b) hydrazine hydrate 99\%, ethanol, heating $10 \mathrm{~h}$; (c) aromatic aldehydes, acetic acid, ethanol 50\%, heating $8 \mathrm{~h}$; (d) chloracetyl chloride, anhydrous 1,4-dioxane, triethylamine, room temperature, stirrer $3 \mathrm{~h}$.

The structure of the compounds was assigned on the basis of spectral (IR, ${ }^{1} \mathrm{H}-\mathrm{NMR},{ }^{13} \mathrm{C}-\mathrm{NMR}$ ) data. The IR spectra of compounds $\mathbf{4} \mathbf{a}_{1-\mathbf{6}}$ (sulfadiazine series) showed absorption bands for the $-\mathrm{CH}_{2}-\mathrm{NH}$ group in the range of $2830-2853 \mathrm{~cm}^{-1}$, for the NH-CO group in the range of 1622-1623 $\mathrm{cm}^{-1}$ and for the characteristic azomethine group $(\mathrm{CH}=\mathrm{N})$ in the $1534-1539 \mathrm{~cm}^{-1}$ range. In the spectra of the sulfisoxazole derivatives $\mathbf{4} \mathbf{b}_{1-6}$ the characteristic absorption bands were observed in the region of $2842-2870 \mathrm{~cm}^{-1}$ (-CH $\left.2-\mathrm{NH}-\right), 1622-1629 \mathrm{~cm}^{-1}$ (NH-CO) and $1507-1540 \mathrm{~cm}^{-1}(\mathrm{CH}=\mathrm{N})$. In the ${ }^{1} \mathrm{H}-\mathrm{NMR}$ spectra of the $\mathrm{N}$-(arylidene)hydrazinoacetyl sulfonamides $\mathbf{4} \mathbf{a}_{1-\mathbf{6}}, \mathbf{4} \mathbf{b}_{\mathbf{1 - 6}}$ the $-\mathrm{CH}_{2}-\mathrm{NH}$ methylene protons resonated as a doublet in the $3.56-3.79 \mathrm{ppm}$ region, while the proton of the azomethine group $(\mathrm{N}=\mathrm{CH})$ 
appeared as a singlet in the 8.06-8.22 ppm region. In the IR spectra of the azetidinone derivatives $\mathbf{5} \mathbf{a}_{\mathbf{1 - 6}}$, $\mathbf{5 b}_{1-6}$ the carbonyl group of the $\beta$-lactam ring appeared as a characteristic absorption band in the range of $1739-1745 \mathrm{~cm}^{-1}$ and $1739-1752 \mathrm{~cm}^{-1}$, respectively. The IR absorption bands and ${ }^{1} \mathrm{H}-\mathrm{NMR}$ signals characteristic of the azomethine group disappeared from the spectra of the azetidinone derivatives, which confirms that the cyclization reaction with chloracetyl chloride took place. The ${ }^{1} \mathrm{H}-\mathrm{NMR}$ spectra of $\mathbf{5} \mathbf{a}_{1-6}$ (sulfadiazine series) and $\mathbf{5} \mathbf{b}_{1-6}$ (sulfisoxazole series) showed two doublets, which are characteristic for $\mathrm{N}-\mathrm{CH}$ and $\mathrm{CH}-\mathrm{Cl}$ that appear in the range of 5.32-5.45 and 5.02-5.23 ppm, respectively. In ${ }^{13} \mathrm{C}$-NMR spectra of the azetidinone derivatives, the characteristic signals for a $\beta$-lactam ring (CH-NH, CH-Cl, $\mathrm{CO}$ cyclic) appeared in the range of 67.8-76.1, 61.04-64.3 and 160.3-162.8 ppm

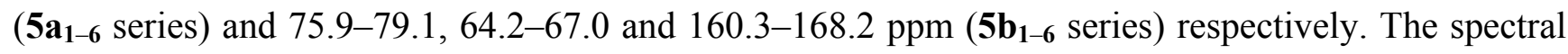
data lend strong support to the proposed structures of all the synthesized compounds.

\subsection{Biological Evaluation}

\subsubsection{Antibacterial Activity}

The antibacterial activities of the title compounds were evaluated using the broth micro-dilution method [24] and the results are listed Tables 1 and 2.

Table 1. MIC values $(\mu \mathrm{g} / \mathrm{mL})$ of the sulfadiazine derivatives $\mathbf{4} \mathbf{a}_{1-6}, \mathbf{5} \mathbf{a}_{1-6}$.

\begin{tabular}{cccccccccc}
\hline \multirow{2}{*}{ Sample e } & \multicolumn{8}{c}{ MICs * values $(\boldsymbol{\mu g} / \mathbf{m L})$} \\
\cline { 2 - 9 } & $\mathbf{S A}$ & $\mathbf{S E}$ & $\mathbf{E F}$ & $\mathbf{K P}$ & $\mathbf{P V}$ & $\mathbf{C F}$ & $\mathbf{E C}$ & $\mathbf{E C}$ & $\mathbf{P A}$ \\
\hline $\mathbf{4 a}_{\mathbf{1}}$ & $>512$ & $>512$ & $>512$ & $>512$ & $>512$ & $>512$ & $>512$ & $>512$ & 256 \\
$\mathbf{4 a}_{\mathbf{2}}$ & $>512$ & 128 & 256 & $>512$ & $>512$ & $>512$ & $>512$ & $>512$ & 128 \\
$\mathbf{4 a}_{\mathbf{3}}$ & $>512$ & $>512$ & $>512$ & $>512$ & $>512$ & $>512$ & $>512$ & $>512$ & $>512$ \\
$\mathbf{4 a}_{\mathbf{4}}$ & $>512$ & $>512$ & $>512$ & $>512$ & $>512$ & $>512$ & $>512$ & $>512$ & 128 \\
$\mathbf{4 a}_{\mathbf{5}}$ & $>512$ & $>512$ & $>512$ & $>512$ & $>512$ & $>512$ & $>512$ & $>512$ & $>512$ \\
$\mathbf{4 a}_{\mathbf{6}}$ & $>512$ & $>512$ & $>512$ & $>512$ & $>512$ & $>512$ & $>512$ & $>512$ & $>512$ \\
$\mathbf{5 a}_{\mathbf{1}}$ & $>512$ & $>512$ & $>512$ & $>512$ & $>512$ & $>512$ & $>512$ & $>512$ & $>512$ \\
$\mathbf{5 a}_{\mathbf{2}}$ & $>512$ & $>512$ & $>512$ & $>512$ & $>512$ & $>512$ & $>512$ & $>512$ & $>512$ \\
$\mathbf{5 a}_{3}$ & $>512$ & $>512$ & $>512$ & $>512$ & $>512$ & $>512$ & $>512$ & $>512$ & $>512$ \\
$\mathbf{5 a}_{\mathbf{4}}$ & $>512$ & $>512$ & $>512$ & $>512$ & $>512$ & $>512$ & $>512$ & $>512$ & $>512$ \\
$\mathbf{5 a}_{\mathbf{5}}$ & $>512$ & $>512$ & $>512$ & $>512$ & $>512$ & $>512$ & $>512$ & $>512$ & $>512$ \\
$\mathbf{5 a}_{\mathbf{6}}$ & $>512$ & $>512$ & $>512$ & $>512$ & $>512$ & $>512$ & $>512$ & $>512$ & $>512$ \\
$\mathbf{S}$ & $>1000$ & $>1000$ & $>1000$ & $>1000$ & $>1000$ & $>1000$ & $>1000$ & $>1000$ & 800 \\
$\mathbf{A}$ & 35.8 & 3 & 3 & 16 & - & - & 8 & 35.8 & 128 \\
\hline
\end{tabular}

* Mean values ( $=3$ ); SA: Staphyloccoccus aureus ATCC 6583; SE: Staphyloccoccus epidermidis ATCC 12228; EF: Enterococcus faecalis ATCC 25912; KP: Klebsiella pneumoniae CIP 53153; PV: Proteus vulgaris CIP 104989; CF: Citrobacter freundii CIP 5732; EC ${ }^{\text {a }}$ : Enterobacter cloacae CIP 103475; EC ' Escherichia coli ATCC 25922; PA: Pseudomonas aeruginosa CIP 82118; S: Sulfanilamide; A: Ampicillin. 
Table 2. MIC values $(\mu \mathrm{g} / \mathrm{mL})$ of the sulfisoxazole derivatives $\mathbf{4} \mathbf{b}_{\mathbf{1 - 6}}, \mathbf{5} \mathbf{b}_{\mathbf{1 - 6}}$.

\begin{tabular}{cccccccccc}
\hline \multirow{2}{*}{ Sample } & \multicolumn{7}{c}{ MICs ${ }^{*}$ values $(\boldsymbol{\mu g} / \mathbf{m L})$} \\
\cline { 2 - 9 } & $\mathbf{S A}$ & $\mathbf{S E}$ & $\mathbf{E F}$ & $\mathbf{K P}$ & $\mathbf{P V}$ & $\mathbf{C F}$ & $\mathbf{E C}^{\mathbf{a}}$ & $\mathbf{E C}$ & $\mathbf{P A}$ \\
\hline $\mathbf{4 b}_{\mathbf{1}}$ & $>512$ & $>512$ & $>512$ & $>512$ & $>512$ & $>512$ & $>512$ & $>512$ & $>512$ \\
$\mathbf{4 b}_{\mathbf{2}}$ & $>512$ & $>512$ & $>512$ & $>512$ & $>512$ & $>512$ & $>512$ & $>512$ & $>512$ \\
$\mathbf{4 b}_{\mathbf{3}}$ & $>512$ & $>512$ & $>512$ & $>512$ & $>512$ & $>512$ & $>512$ & $>512$ & $>512$ \\
$\mathbf{4 b}_{\mathbf{4}}$ & $>512$ & $>512$ & $>512$ & $>512$ & $>512$ & $>512$ & $>512$ & $>512$ & $>512$ \\
$\mathbf{4} \mathbf{b}_{\mathbf{5}}$ & $>512$ & $>512$ & $>512$ & $>512$ & $>512$ & $>512$ & $>512$ & $>512$ & 256 \\
$\mathbf{4} \mathbf{b}_{\mathbf{6}}$ & $>512$ & $>512$ & $>512$ & $>512$ & $>512$ & $>512$ & $>512$ & $>512$ & $>512$ \\
$\mathbf{5 b}_{\mathbf{1}}$ & $>512$ & $>512$ & $>512$ & $>512$ & $>512$ & $>512$ & $>512$ & $>512$ & $>512$ \\
$\mathbf{5} \mathbf{b}_{\mathbf{2}}$ & $>512$ & $>512$ & $>512$ & $>512$ & $>512$ & $>512$ & $>512$ & $>512$ & $>512$ \\
$\mathbf{5} \mathbf{b}_{\mathbf{3}}$ & $>512$ & $>512$ & $>512$ & $>512$ & $>512$ & $>512$ & $>512$ & $>512$ & $>512$ \\
$\mathbf{5} \mathbf{b}_{\mathbf{4}}$ & $>512$ & $>512$ & $>512$ & $>512$ & $>512$ & $>512$ & $>512$ & $>512$ & $>512$ \\
$\mathbf{5} \mathbf{b}_{\mathbf{5}}$ & $>512$ & $>512$ & $>512$ & $>512$ & $>512$ & $>512$ & $>512$ & $>512$ & $>512$ \\
$\mathbf{5} \mathbf{b}_{\mathbf{6}}$ & $>512$ & $>512$ & $>512$ & $>512$ & $>512$ & $>512$ & $>512$ & $>512$ & $>512$ \\
$\mathbf{S}$ & $>1000$ & $>1000$ & $>1000$ & $>1000$ & $>1000$ & $>1000$ & $>1000$ & $>1000$ & 800 \\
$\mathbf{A}$ & 35.8 & 3 & 3 & 16 & - & - & 8 & 35.8 & 128 \\
\hline
\end{tabular}

* Mean values $(\mathrm{n}=3)$; SA: Staphyloccoccus aureus ATCC 6583; SE: Staphyloccoccus epidermidis ATCC 12228; EF: Enterococcus faecalis ATCC 25912; KP: Klebsiella pneumoniae CIP 53153; PV: Proteus vulgaris CIP 104989; CF: Citrobacter freundii CIP 5732; EC ${ }^{\text {a }: ~ E n t e r o b a c t e r ~ c l o a c a e ~ C I P ~ 103475 ; ~ E C ~}{ }^{\text {b: }}$ Escherichia coli ATCC 25922; PA: Pseudomonas aeruginosa CIP 82118; S: Sulfanilamide; A: Ampicillin.

The minimum inhibitory concentrations (MICs) of almost all compounds were more than $512 \mu \mathrm{g} / \mathrm{mL}$. The $N$-(arylidene)hydrazinoacetyl derivative of sulfadiazine (compound $\mathbf{4} \mathbf{a}_{2}$ ) was the most active compound, as it was active on Staphyloccoccus epidermidis ATCC 12228 (128 $\mu \mathrm{g} / \mathrm{mL})$, Enterococcus faecalis ATCC $25912(256 \mu \mathrm{g} / \mathrm{mL})$ and Pseudomonas aeruginosa CIP $82118(128 \mu \mathrm{g} / \mathrm{mL})$. The compounds $\mathbf{4} \mathbf{a}_{\mathbf{1}}(256 \mu \mathrm{g} / \mathrm{mL}), \mathbf{4} \mathbf{a}_{\mathbf{4}}(128 \mu \mathrm{g} / \mathrm{mL})$ and $\mathbf{4} \mathbf{b}_{\mathbf{5}}(256 \mu \mathrm{g} / \mathrm{mL})$ were active against Pseudomonas aeruginosa. All tested compounds are more active than sulfanilamide, but less active than ampicillin used as positive controls.

\subsubsection{Antioxidant Activity}

\subsubsection{Ferric Reducing Power}

The measurement of reducing power defines an important aspect of the antioxidant activity of the compounds. In this assay, the presence of a reducing agent in the sample results in reducing of the ferric/ferricyanide complex to its ferrous $\left(\mathrm{Fe}^{2+}\right)$ form. The amount of $\mathrm{Fe}^{2+}$ is then quantitatively monitored by measuring the intensity of Perl's Prussian blue colour complex at $695 \mathrm{~nm}$ [25]. The results expressed as $\mathrm{EC}_{50}$ values $(\mathrm{mg} / \mathrm{mL})$ are presented in Tables 3 and 4 . The small value of the $\mathrm{EC}_{50}$ indicates a higher ferric reducing power. 
Table 3. Ferric reducing power $\left(\mathrm{EC}_{50} \mathrm{mg} / \mathrm{mL}\right)$ of the sulfadiazine derivatives $\mathbf{4} \mathbf{a}_{1-6}, \mathbf{5} \mathbf{a}_{1-6}$.

\begin{tabular}{cccc}
\hline Sample & $\mathbf{E C}_{\mathbf{5 0}} \mathbf{~ m g} / \mathbf{m L}$ & Sample & $\mathbf{E C}_{\mathbf{5 0}} \mathbf{~ m g} / \mathbf{m L}$ \\
\hline $\mathbf{4 a}_{\mathbf{1}}$ & $0.0663 \pm 0.0056$ & $\mathbf{5 a}_{\mathbf{1}}$ & $0.1553 \pm 0.0152$ \\
$\mathbf{4 a}_{\mathbf{2}}$ & $0.0756 \pm 0.0040$ & $\mathbf{5 a}_{\mathbf{2}}$ & $0.1376 \pm 0.0002$ \\
$\mathbf{4 a}_{3}$ & $0.0856 \pm 0.0051$ & $\mathbf{5 a}_{3}$ & $0.1745 \pm 0.0125$ \\
$\mathbf{4 a}_{\mathbf{4}}$ & $0.0790 \pm 0.0026$ & $\mathbf{5 a}_{\mathbf{4}}$ & $0.1798 \pm 0.0018$ \\
$\mathbf{4 a}_{\mathbf{5}}$ & $0.0510 \pm 0.0036$ & $\mathbf{5 a}_{\mathbf{5}}$ & $0.0945 \pm 0.0085$ \\
$\mathbf{4 a}_{\mathbf{6}}$ & $0.0503 \pm 0.0025$ & $\mathbf{5 a}_{\mathbf{6}}$ & $0.2277 \pm 0.0037$ \\
$\mathbf{1 a}$ & $2.6140 \pm 0.0301$ & $\mathbf{A A}$ & $0.0075 \pm 0.0002$ \\
\hline
\end{tabular}

Data are mean $\pm \mathrm{SD}(\mathrm{n}=3, p<0.05)$.

As it can be seen both $N$-(arylidene)hydrazinoacetyl and azetidinone derivatives are more active than their sulfonamide parents, sulfadiazine (1a) and sulfizoxazole (1b). In the $N$-(arylidene) hydrazinoacetyl series of sulfadiazine (compounds 4a-6) it was observed that the most active compounds were those which resulted from reaction of condensation with 4-hydroxybenzaldehyde (compound $\mathbf{4} \mathbf{a}_{5}$ ) and 4-nitrobenzaldehyde (compound $\mathbf{4 a}_{\mathbf{6}}$ ). The values of $\mathrm{EC}_{50}$ for these compounds were $0.0510 \pm 0.0036$ (compound $\mathbf{4 \mathbf { a } _ { 5 }}$ ) and $0.0503 \pm 0.0025$ (compound $\mathbf{4} \mathbf{a}_{\mathbf{6}}$ ), which means that they are about 50 times more active than sulfadiazine $\left(\mathrm{EC}_{50}=2.6140 \pm 0.0301\right)$. Concerning the azetidinone series the most active compound was $\mathbf{5} \mathbf{a}_{5}$, which is the analogue of $\mathbf{4} \mathbf{a}_{5}$ in the azetidinone series. This compound is approximately 28 time more active $\left(\mathrm{EC}_{50}=0.0945 \pm 0.0085\right)$ then sulfadiazine $\left(\mathrm{EC}_{50}=2.6140 \pm 0.0301\right)$ (Table 3$)$. The ferric reducing power of the compounds resulting through modulation of sulfisoxazole is lower than that of the the analogues of the sulfadiazine series. In reference to sulfisoxazole (1b), all tested compounds $\mathbf{4} \mathbf{b}_{\mathbf{1 - 6}}, \mathbf{5} \mathbf{b}_{\mathbf{1 - 6}}$ are more active. The most active compounds were $\mathbf{4} \mathbf{b}_{\mathbf{5}}$ [ $N$-(arylidene)hydrazinoacetyl series] and $\mathbf{5} \mathbf{b}_{\mathbf{5}}$ (azetidinone series), which have in their structure the 4-hydroxyphenyl radical. These compounds are 46 times $\left(\mathbf{4 b}_{\mathbf{5}}, \mathrm{EC}_{50}=0.0210 \pm 0.0065\right)$ and 10 times $\left(\mathbf{5 b}_{5}, \mathrm{EC}_{50}=0.0935 \pm 0.0098\right)$ more active, respectively, than sulfisoxazole (1b, $\left.\mathrm{EC}_{50}=0.9640 \pm 0.0443\right)$ (Table 4).

Table 4. Ferric reducing power $\left(\mathrm{EC}_{50} \mathrm{mg} / \mathrm{mL}\right)$ of the sulfisoxazole derivatives $\mathbf{4} \mathbf{b}_{\mathbf{1 - 6}}, \mathbf{5} \mathbf{b}_{\mathbf{1 - 6}}$.

\begin{tabular}{cccc}
\hline Sample & $\mathbf{E C}_{\mathbf{5 0}} \mathbf{~} \mathbf{m g} / \mathbf{m L}$ & Sample & $\mathbf{E C}_{\mathbf{5 0}} \mathbf{~ m g} / \mathbf{m L}$ \\
\hline $\mathbf{4 b}_{\mathbf{1}}$ & $0.0756 \pm 0.0055$ & $\mathbf{5 b}_{\mathbf{1}}$ & $0.1450 \pm 0.0003$ \\
$\mathbf{4 b}_{\mathbf{2}}$ & $0.2043 \pm 0.0055$ & $\mathbf{5 b}_{\mathbf{2}}$ & $0.1164 \pm 0.0025$ \\
$\mathbf{4 b}_{\mathbf{3}}$ & $0.0610 \pm 0.0036$ & $\mathbf{5 b}_{\mathbf{3}}$ & $0.1915 \pm 0.0216$ \\
$\mathbf{4 b}_{\mathbf{4}}$ & $0.0612 \pm 0.0040$ & $\mathbf{5 b}_{\mathbf{4}}$ & $0.3182 \pm 0.0411$ \\
$\mathbf{4 b}_{\mathbf{5}}$ & $0.0210 \pm 0.0065$ & $\mathbf{5 b}_{\mathbf{5}}$ & $0.0935 \pm 0.0098$ \\
$\mathbf{4 b}_{\mathbf{6}}$ & $0.1173 \pm 0.0066$ & $\mathbf{5} \mathbf{b}_{\mathbf{6}}$ & $0.1106 \pm 0.0149$ \\
$\mathbf{1 b}$ & $0.9640 \pm 0.0443$ & $\mathbf{A A}$ & $0.0075 \pm 0.0002$ \\
\hline
\end{tabular}

Data are mean $\pm \mathrm{SD}(\mathrm{n}=3, p<0.05)$.

The chemical modulation of the parent sulfonamides improve their ferric reducing power and all tested compounds are more active than sulfadiazine and sulfisoxazole, respectively, but they are less active than ascorbic acid (AA) at the same concentration. 


\subsubsection{Total Antioxidant Activity}

The total antioxidant activity was determined using phophomolybdenum blue complex with a maximum absorption at $695 \mathrm{~nm}$ [26]. The data presented in Tables 5 and 6 show that the tested compounds are more active than sulfadiazine and sulfisoxazole, respectively, and moreover, the sulfadiazine derivatives are more active than sulfisoxazole compounds.

Table 5. Total antioxidant activity $\left(\mathrm{EC}_{50} \mathrm{mg} / \mathrm{mL}\right)$ of the sulfadiazine derivatives $\mathbf{4} \mathbf{a}_{1-6}, \mathbf{5} \mathbf{a}_{1-6}$.

\begin{tabular}{cccc}
\hline Sample & $\mathbf{E C}_{\mathbf{5 0}} \mathbf{~ m g} / \mathbf{m L}$ & Sample & $\mathbf{E C}_{\mathbf{5 0}} \mathbf{~ m g} / \mathbf{m L}$ \\
\hline $\mathbf{4 a}_{\mathbf{1}}$ & $0.0180 \pm 0.0044$ & $\mathbf{5 a}_{\mathbf{1}}$ & $0.0398 \pm 0.0022$ \\
$\mathbf{4 a}_{\mathbf{2}}$ & $0.0280 \pm 0.0067$ & $\mathbf{5 a}_{\mathbf{2}}$ & $0.0498 \pm 0.0015$ \\
$\mathbf{4 a}_{\mathbf{3}}$ & $0.0110 \pm 0.0007$ & $\mathbf{5 a}_{\mathbf{3}}$ & $0.0330 \pm 0.0098$ \\
$\mathbf{4 a}_{\mathbf{4}}$ & $0.0360 \pm 0.0089$ & $\mathbf{5 a}_{\mathbf{4}}$ & $0.0507 \pm 0.0037$ \\
$\mathbf{4 a}_{\mathbf{5}}$ & $0.0440 \pm 0.0050$ & $\mathbf{5 a}_{\mathbf{5}}$ & $0.0563 \pm 0.0009$ \\
$\mathbf{4 a}_{\mathbf{6}}$ & $0.0220 \pm 0.0072$ & $\mathbf{5} \mathbf{a}_{\mathbf{6}}$ & $0.0341 \pm 0.0055$ \\
$\mathbf{1 a}$ & $6.6483 \pm 0.0180$ & $\mathbf{A A}$ & $0.0067 \pm 0.0003$ \\
\hline \multicolumn{4}{c}{ Data are mean $\pm \mathrm{SD}(\mathrm{n}=3, p<0.05)}$.
\end{tabular}

Table 6. Total antioxidant activity $\left(\mathrm{EC}_{50} \mathrm{mg} / \mathrm{mL}\right)$ of the sulfisoxazole derivatives $\mathbf{4} \mathbf{b}_{1-6}, \mathbf{5 b}_{1-\mathbf{6}}$.

\begin{tabular}{cccc}
\hline Sample & $\mathbf{E C 5 0} \mathbf{~ m g} / \mathbf{m L}$ & Sample & $\mathbf{E C 5 0} \mathbf{~ m g} / \mathbf{m L}$ \\
\hline $\mathbf{4} \mathbf{b}_{\mathbf{1}}$ & $0.0481 \pm 0.0042$ & $\mathbf{5 b}_{\mathbf{1}}$ & $0.0433 \pm 0.0009$ \\
$\mathbf{4} \mathbf{b}_{\mathbf{2}}$ & $0.0612 \pm 0.0078$ & $\mathbf{5 b}_{\mathbf{2}}$ & $0.0756 \pm 0.0033$ \\
$\mathbf{4 b}_{\mathbf{3}}$ & $0.0330 \pm 0.0009$ & $\mathbf{5 b}_{\mathbf{3}}$ & $0.0574 \pm 0.0025$ \\
$\mathbf{4 b}_{\mathbf{4}}$ & $0.0332 \pm 0.0047$ & $\mathbf{5 b}_{\mathbf{4}}$ & $0.0385 \pm 0.0078$ \\
$\mathbf{4 b}_{\mathbf{5}}$ & $0.0551 \pm 0.0086$ & $\mathbf{5 b}_{\mathbf{5}}$ & $0.0718 \pm 0.0008$ \\
$\mathbf{4} \mathbf{b}_{\mathbf{6}}$ & $0.0794 \pm 0.0091$ & $\mathbf{5} \mathbf{b}_{\mathbf{6}}$ & $0.0825 \pm 0.0045$ \\
$\mathbf{1 b}$ & $21.658 \pm 0.0224$ & $\mathbf{A A}$ & $0.0067 \pm 0.0003$ \\
\hline \multicolumn{4}{c}{ Data are mean $\pm \mathrm{SD}(\mathrm{n}=3, p<0.05)}$.
\end{tabular}

The most favorable influence seems to be the presence of halogen on the phenyl ring, especially the presence of chlorine in the sulfadiazine series (compounds $\mathbf{4} \mathbf{a}_{3}, \mathbf{5} \mathbf{a}_{3}$ ) and the presence of chlorine and bromine in the sulfisoxazole series (compounds $\left.\mathbf{4 b}_{\mathbf{3}-\mathbf{4}}, \mathbf{5 b}_{\mathbf{3}-\mathbf{4}}\right)$. The compound $\mathbf{4 a}_{\mathbf{3}}\left(\mathrm{EC}_{50}=0.0110 \pm 0.0007\right.$ ) is about 600 times more active than sulfadiazine (1a) $\left(\mathrm{EC}_{50}=6.6483 \pm 0.0180\right)$ and its antioxidant activity is comparable with the activity of ascorbic acid (AA) $\left(\mathrm{EC}_{50}=0.0067 \pm 0.0003\right)$. Although its azetidinone analogue $\mathbf{5} \mathbf{a}_{3}$ has a lower activity, it remains significant in reference with sulfadiazine (Table 5). In the sulfisoxazole series the compounds $\mathbf{4 b}_{\mathbf{3}}\left(\mathrm{EC}_{50}=0.0330 \pm 0.0009\right)$ and $\mathbf{4 b}_{\mathbf{4}}$ $\left(\mathrm{EC}_{50}=0.0332 \pm 0.0047\right)$ are approximately 650 time more active than sulfisoxazole (1b) $\left(\mathrm{EC}_{50}=21.658 \pm 0.0224\right)$. Their azetidinone analogues are 380 times $\left(\mathbf{5} \mathbf{b}_{\mathbf{3}}, \mathrm{EC}_{50}=0.0574 \pm 0.0025\right)$ and 560 times $\left(\mathbf{5 b}_{\mathbf{4}}, \mathrm{EC}_{50}=0.0385 \pm 0.0078\right)$ more active than sulfisoxazole.

\subsubsection{DPPH Radical Scavenging Assay}

DPPH is a well-know radical which demonstrates a strong absorption band centered at about $517 \mathrm{~nm}$, and it becomes colorless or pale yellow when it is neutralized. DPPH radical is scavenged by 
antioxidants through the donation of proton forming the reduced DPPH, and it is commonly used to evaluate the radical scavenging capacity of antioxidants [27]. The scavenging activities of the $N$-(arylidene)hydrazinoacetyl sulfonamides $\quad \mathbf{4 a _ { 1 - 6 }}, \quad \mathbf{4} \mathbf{b}_{1-6}$ and $N$-(4-aryl-3-chloro-2-oxoazetidin-1-

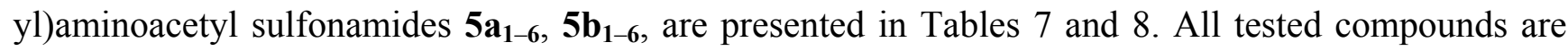
more active than their parent (sulfadiazine and sulfisoxazole) sulfonamides and some of them have a scavenging ability comparable with the scavenging ability of ascorbic acid.

Table 7. DPPH radical scavenging ability of sulfadiazine derivatives $\mathbf{4} \mathbf{a}_{1-6}, \mathbf{5} \mathbf{a}_{1-6}$.

\begin{tabular}{cccc}
\hline Sample & Scavenging ability (\%) & Sample & Scavenging ability (\%) \\
\hline $\mathbf{4 a}_{\mathbf{1}}$ & $87.73 \pm 0.69$ & $\mathbf{5 a}_{\mathbf{1}}$ & $67.18 \pm 0.14$ \\
$\mathbf{4 a}_{\mathbf{2}}$ & $92.22 \pm 0.89$ & $\mathbf{5 a}_{\mathbf{2}}$ & $78.25 \pm 0.49$ \\
$\mathbf{4 a}_{3}$ & $73.09 \pm 0.50$ & $\mathbf{5 a}_{3}$ & $80.94 \pm 0.74$ \\
$\mathbf{4 a}_{\mathbf{4}}$ & $85.66 \pm 0.89$ & $\mathbf{5 a}_{4}$ & $71.52 \pm 0.48$ \\
$\mathbf{4 a}_{\mathbf{5}}$ & $93.17 \pm 0.64$ & $\mathbf{5 a}_{\mathbf{5}}$ & $61.28 \pm 0.13$ \\
$\mathbf{4 a}_{\mathbf{6}}$ & $77.28 \pm 0.83$ & $\mathbf{5 a}$ & $71.61 \pm 0.33$ \\
$\mathbf{1 a}$ & $11.15 \pm 0.24$ & $\mathbf{A A}$ & $97.08 \pm 0.52$ \\
\hline
\end{tabular}

Table 8. DPPH radical scavenging ability of sulfisoxazole derivatives $\mathbf{4} \mathbf{b}_{\mathbf{1 - 6}}, \mathbf{5} \mathbf{b}_{\mathbf{1 - 6}}$.

\begin{tabular}{cccc}
\hline Sample & Scavenging ability (\%) & Sample & Scavenging ability (\%) \\
\hline $\mathbf{4 b}_{\mathbf{1}}$ & $48.17 \pm 0.63$ & $\mathbf{5 b}_{\mathbf{1}}$ & $64.28 \pm 0.49$ \\
$\mathbf{4 b}_{\mathbf{2}}$ & $6.43 \pm 0.41$ & $\mathbf{5 b}_{\mathbf{2}}$ & $44.03 \pm 0.13$ \\
$\mathbf{4 b}_{\mathbf{3}}$ & $67.39 \pm 0.52$ & $\mathbf{5 b}_{\mathbf{3}}$ & $44.01 \pm 0.86$ \\
$\mathbf{4 b}_{\mathbf{4}}$ & $42.95 \pm 0.23$ & $\mathbf{5 b}_{\mathbf{4}}$ & $35.52 \pm 0.48$ \\
$\mathbf{4 b}_{\mathbf{5}}$ & $61.20 \pm 0.68$ & $\mathbf{5 b}_{\mathbf{5}}$ & $61.28 \pm 0.13$ \\
$\mathbf{4 b}_{\mathbf{6}}$ & $82.65 \pm 0.18$ & $\mathbf{5} \mathbf{b}_{\mathbf{6}}$ & $33.81 \pm 0.09$ \\
$\mathbf{1 b}$ & $36.59 \pm 0.08$ & $\mathbf{A A}$ & $97.08 \pm 0.52$ \\
\hline
\end{tabular}

The compounds obtained starting from sulfadiazine (compounds $\mathbf{4} \mathbf{a}_{1-6}, \mathbf{5} \mathbf{a}_{1-6}$, Table 7) are more active than sulfisoxazole derivatives $\mathbf{4} \mathbf{b}_{1-\mathbf{6}}, \mathbf{5} \mathbf{b}_{\mathbf{1 - 6}}$ (Table 8). In reference with sulfadiazine (1a), its $N$-(arylidene)hydrazinoacetyl derivatives $\mathbf{4} \mathbf{a}_{1-6}$ are 6.5-8.4 times more active. Under similar conditions the azetidinone derivatives $\mathbf{5} \mathbf{a}_{1-6}$ are slightly less active, being 5.5-7.3 more active than sulfadiazine. The most active compound is $\mathbf{4} \mathbf{a}_{\mathbf{5}}$ [ $N^{4}$-(4-hydroxybenzylidene)hydrazinoacetylamino- $N^{1}$-(pyrimidin-2yl)benzensulfonamide]; its scavenging ability $(93.17 \pm 0.64)$ being 8.4 time higher than sulfadiazine $(11.15 \pm 0.24)$ and comparable with ascorbic acid $(97.08 \pm 0.52)$.

\section{Experimental}

\subsection{General Procedures}

Melting points were measured using a Buchi Melting Point B-540 apparatus and are uncorrected. The FT-IR spectra were recorded on an ABB Bomen MB3000 spectrometer, over a $500-4000 \mathrm{~cm}^{-1}$ range, after 32 scans at a resolution of $4 \mathrm{~cm}^{-1}$. The spectra processing was carried out with Horizon $\mathrm{MB}^{\mathrm{TM}}$ FTIR Software. The ${ }^{1} \mathrm{H}-\mathrm{NMR}(300 \mathrm{MHz})$ and ${ }^{13} \mathrm{C}-\mathrm{NMR}(75 \mathrm{MHz})$ spectra were obtained on a Bruker Avance ARX-300 spectrometer using tetramethylsilane as internal standard and DMSO- $d_{6}$ as 
solvent. The chemical shifts are shown in $\delta$ values $(\mathrm{ppm})$. The progress of the reaction was monitored on TLC, using pre-coated Kieselgel 60 F254 plates (Merck) and the compounds were visualized by UV light.

\subsection{Synthetic Procedures}

\subsubsection{Preparation of $N$-(arylidene)hydrazinoacetyl Sulfonamides $\mathbf{4} \mathbf{a}_{1-\mathbf{6}} ; \mathbf{4} \mathbf{b}_{\mathbf{1 - 6}}$}

To a solution of hydrazinoacetyl sulfonamide derivatives $(10 \mathrm{mmol})$ in ethanol $50 \%(200 \mathrm{~mL})$, glacial acetic acid $(0.5 \mathrm{~mL})$ and the appropriate aldehyde $(10 \mathrm{mmol})$ were added. The mixture was heated under reflux for $8 \mathrm{~h}$, and then it was cooled at room temperature. The solid was filtered off, dried and recrystallized from isopropyl alcohol.

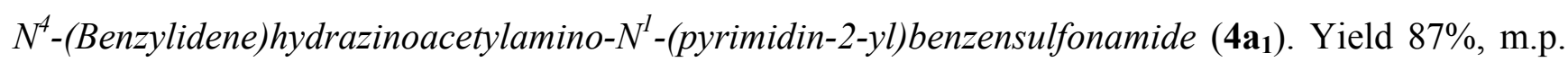
200-202 ${ }^{\circ} \mathrm{C}$; IR ( $\left.\mathrm{KBr}, \mathrm{cm}^{-1}\right): 3450(-\mathrm{NH}), 2853\left(\mathrm{CH}_{2}-\mathrm{NH}\right), 2924$ (=CH- pyrimidine ring), 1693 $(\mathrm{C}=\mathrm{O}), 1623(\mathrm{HN}-\mathrm{CO}), 1591(-\mathrm{C}=\mathrm{C}-$ aromatic ring), $1534(\mathrm{~N}=\mathrm{CH}), 1448(-\mathrm{C}=\mathrm{C}$ - pyrimidine ring), 1313 (C-O), 1128 (-NH-SO $), 1097$ (CH aromatic ring), 947 (S-N), 840 (S-C); ${ }^{1} \mathrm{H}-\mathrm{NMR}$ 8: 8.72-8.91 (dm, 3H, pyrimidine ring), $8.27(\mathrm{~s}, 1 \mathrm{H}, \mathrm{CO}-\mathrm{NH}), 8.08(\mathrm{~s}, 1 \mathrm{H}, \mathrm{N}=\mathrm{CH}), 7.15-7.90(\mathrm{dm}, 9 \mathrm{H}, A r-\mathrm{H}), 4.19$ (s, $\left.1 \mathrm{H},-\mathrm{NH}-\mathrm{SO}_{2}\right), 3.60\left(\mathrm{~d}, 2 \mathrm{H}, \mathrm{CH}_{2}\right), 3.43(\mathrm{~m}, 1 \mathrm{H}, \mathrm{HN}-\mathrm{N})$.

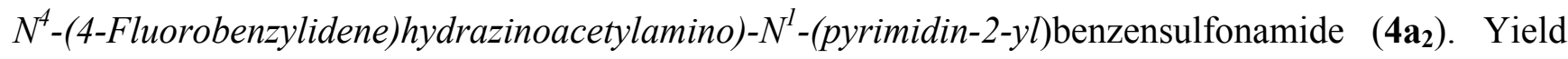
90\%, m.p. $20-210^{\circ} \mathrm{C}$; IR (KBr, cm $\left.{ }^{-1}\right): 3350(-\mathrm{NH}), 2833\left(\mathrm{CH}_{2}-\mathrm{NH}\right), 2933$ (=CH- pyrimidine ring), $1693(\mathrm{C}=\mathrm{O}), 1622(\mathrm{HN}-\mathrm{CO}), 1592(-\mathrm{C}=\mathrm{C}$ - aromatic ring), $1535(\mathrm{~N}=\mathrm{CH}), 1455(-\mathrm{C}=\mathrm{C}-$ pyrimidine ring), 1314 (C-O), 1229 (C-F), 1130 (-NH-SO ${ }_{2}$ ), 1099 (CH aromatic ring), 928 (S-N), 836 (S-C); ${ }^{1} \mathrm{H}-\mathrm{NMR}$ $\delta$ : $8.63-8.90(\mathrm{dm}, 3 \mathrm{H}$, pyrimidine ring), $8.29(\mathrm{~s}, 1 \mathrm{H}, \mathrm{CO}-\mathrm{NH}), 8.10(\mathrm{~s}, 1 \mathrm{H}, \mathrm{N}=\mathrm{CH}), 7.1-7.95(\mathrm{~d}, 8 \mathrm{H}, A r-\mathrm{H})$, 4.20 (s, 1H, -NH-SO 2 ), 3.79 (d, 2H, $\left.\mathrm{CH}_{2}\right), 3.45$ (m, 1H, HN-N).

$N^{4}$-(4-Chlorobenzylidene)hydrazinoacetylamino- $N^{l}$-(pyrimidin-2-yl)benzensulfonamide $\quad\left(\mathbf{4} \mathbf{a}_{3}\right)$. Yield 83\% m.p. $20-207{ }^{\circ} \mathrm{C}$; IR $\left(\mathrm{KBr}, \mathrm{cm}^{-1}\right): 3448(-\mathrm{NH}), 2830\left(\mathrm{CH}_{2}-\mathrm{NH}\right), 2930(=\mathrm{CH}-$ pyrimidine ring), $1695(\mathrm{C}=\mathrm{O}), 1623(\mathrm{HN}-\mathrm{CO}), 1591(-\mathrm{C}=\mathrm{C}$ - aromatic ring), $1536(\mathrm{~N}=\mathrm{CH}), 1496(-\mathrm{C}=\mathrm{C}-$ pyrimidine ring), 1312 (C-O), $1173\left(-\mathrm{NH}-\mathrm{SO}_{2}\right), 1084(\mathrm{CH}$ aromatic ring), 940 (S-N), 839 (S-C), 751 (C-Cl); ${ }^{1} \mathrm{H}-\mathrm{NMR}$ : $8.7-8.89(\mathrm{dm}, 3 \mathrm{H}$, pyrimidine ring), $8.22(\mathrm{~s}, 1 \mathrm{H}, \mathrm{CO}-\mathrm{NH}), 8.15(\mathrm{~s}, 1 \mathrm{H}, \mathrm{N}=\mathrm{CH}), 7.0-7.76$ (d, $8 \mathrm{H}, A r-\mathrm{H}), 4.17$ (s, 1H, -NH-SO 2 ), 3.61 (d, 2H, $\left.\mathrm{CH}_{2}\right), 3.40$ (m, 1H, HN-N).

$N^{4}$-(4-Bromobenzylidene)hydrazinoacetylamino- $N^{1}$-(pyrimidin-2-yl)benzensulfonamide $\quad\left(\mathbf{4} \mathbf{a}_{4}\right)$. Yield 81\%, m.p. $212-213{ }^{\circ} \mathrm{C}$; IR ( $\left.\mathrm{KBr}, \mathrm{cm}^{-1}\right): 3446(-\mathrm{NH}), 2850\left(\mathrm{CH}_{2}-\mathrm{NH}\right), 2940(=\mathrm{CH}-$ pyrimidine ring), $1690(\mathrm{C}=\mathrm{O}), 1623(\mathrm{HN}-\mathrm{CO}), 1591(-\mathrm{C}=\mathrm{C}$ - aromatic ring), $1535(\mathrm{~N}=\mathrm{CH}), 1454(-\mathrm{C}=\mathrm{C}-$ pyrimidine ring), 1314 (C-O), 1130 (-NH-SO ${ }_{2}$ ), 1079 (CH aromatic ring), 959 (S-N), 840 (S-C), 550 (C-Br); ${ }^{1} \mathrm{H}-\mathrm{NMR}$ $\delta$ : $8.71-8.89(\mathrm{dm}, 3 \mathrm{H}$, pyrimidine ring), $8.21(\mathrm{~s}, 1 \mathrm{H}, \mathrm{CO}-\mathrm{NH}), 8.18(\mathrm{~s}, 1 \mathrm{H}, \mathrm{N}=\mathrm{CH}), 7.19-7.84(\mathrm{~d}, 8 \mathrm{H}$, $A r-\mathrm{H}), 4.16\left(\mathrm{~s}, 1 \mathrm{H},-\mathrm{NH}-\mathrm{SO}_{2}\right), 3.56\left(\mathrm{~d}, 2 \mathrm{H}, \mathrm{CH}_{2}\right), 3.40(\mathrm{~m}, 1 \mathrm{H}, \mathrm{HN}-\mathrm{N})$.

$N^{4}$-(4-Hydroxybenzylidene)hydrazinoacetylamino- $N^{l}$-(pyrimidin-2-yl)benzensulfonamide (4a5). Yield 86\%, m.p. 208-209 ${ }^{\circ} \mathrm{C}$; IR ( $\left.\mathrm{KBr}, \mathrm{cm}^{-1}\right): 3505(\mathrm{OH}), 3335(-\mathrm{NH}), 2850\left(\mathrm{CH}_{2}-\mathrm{NH}\right), 2932(=\mathrm{CH}-$ pyrimidine ring), $1677(\mathrm{C}=\mathrm{O}), 1622$ (HN-CO), $1590(-\mathrm{C}=\mathrm{C}-$ aromatic ring), $1539(\mathrm{~N}=\mathrm{CH}), 1514$ 
(-C=C- pyrimidine ring), 1304 (C-O), 1168 (-NH-SO $), 1078$ (CH aromatic ring), 965 (S-N), 839 (S-C); ${ }^{1} \mathrm{H}-\mathrm{NMR} \delta$ : 8.56-8.89 (dm, 3H, pyrimidine ring), $8.24(\mathrm{~s}, 1 \mathrm{H}, \mathrm{CO}-\mathrm{NH}), 8.07(\mathrm{~s}, 1 \mathrm{H}, \mathrm{N}=\mathrm{CH}), 7.35-7.89$ (d, $8 \mathrm{H}, A r-\mathrm{H}), 5.05$ (s, 1H, Ar-OH), 4.17 (s, 1H, -NH-SO $), 3.63$ (d, 2H, $\left.\mathrm{CH}_{2}\right), 3.44$ (m, 1H, HN-N).

$N^{4}$-(4-Nitrobenzylidene)hydrazinoacetylamino- $N^{1}$-(pyrimidin-2-yl)benzensulfonamide $\left(\mathbf{4 a}_{\mathbf{6}}\right)$. Yield 75\%, m.p. $227-230{ }^{\circ} \mathrm{C}$; IR $\left(\mathrm{KBr}, \mathrm{cm}^{-1}\right)$ : $3445(-\mathrm{NH}), 2851\left(\mathrm{CH}_{2}-\mathrm{NH}\right), 2932$ (=CH- pyrimidine ring), 1689 $(\mathrm{C}=\mathrm{O}), 1623(\mathrm{HN}-\mathrm{CO}), 1592(-\mathrm{C}=\mathrm{C}$ - aromatic ring), $1536(\mathrm{~N}=\mathrm{CH}), 1514(-\mathrm{C}=\mathrm{C}$ - pyrimidine ring), 1343 $\left(\mathrm{C}-\mathrm{NO}_{2}\right), 1316(\mathrm{C}-\mathrm{O}), 1174\left(-\mathrm{NH}-\mathrm{SO}_{2}\right), 1078\left(\mathrm{CH}\right.$ aromatic ring), $951(\mathrm{~S}-\mathrm{N}), 837(\mathrm{~S}-\mathrm{C}) ;{ }^{1} \mathrm{H}-\mathrm{NMR} \delta$ : 8.50-8.87 (dm, 3H, pyrimidine ring), 8.21 (s, 1H, CO-NH), $8.06(\mathrm{~s}, 1 \mathrm{H}, \mathrm{N}=\mathrm{CH}), 7.33-7.90(\mathrm{~d}, 8 \mathrm{H}, A r-\mathrm{H})$, 4.16 (s, $\left.1 \mathrm{H},-\mathrm{NH}-\mathrm{SO}_{2}\right), 3.70$ (d, 2H, $\left.\mathrm{CH}_{2}\right), 3.41$ (m, 1H, HN-N).

$N^{4}$-(Benzylidene)hydrazinoacetylamino- $N^{1}$-(3,4-dimethyl-1,2-oxazol-5-yl)benzensulfonamide (4) $\mathbf{4}$ ). Yield 87\%, m.p. $118-120{ }^{\circ} \mathrm{C}$; IR (KBr, cm $\left.{ }^{-1}\right): 3465(-\mathrm{NH}), 2864\left(\mathrm{CH}_{2}-\mathrm{NH}\right), 1686(\mathrm{C}=\mathrm{O}), 1625(\mathrm{HN}-\mathrm{CO})$, $1592\left(-\mathrm{C}=\mathrm{C}\right.$ - aromatic ring), $1518(\mathrm{~N}=\mathrm{CH}), 1496\left(-\mathrm{C}=\mathrm{C}\right.$ - oxazole ring), $1310(\mathrm{C}-\mathrm{O}), 1151\left(-\mathrm{NH}-\mathrm{SO}_{2}\right)$, $1093\left(\mathrm{CH}\right.$ aromatic ring), $956(\mathrm{~S}-\mathrm{N}), 830(\mathrm{~S}-\mathrm{C}) ;{ }^{1} \mathrm{H}-\mathrm{NMR} \delta: 8.41(\mathrm{~s}, 1 \mathrm{H}, \mathrm{CO}-\mathrm{NH}), 8.12(\mathrm{~s}, 1 \mathrm{H}$, $\mathrm{N}=\mathrm{CH}), 7.21-7.93(\mathrm{dm}, 9 \mathrm{H}, A r-\mathrm{H}), 4.62\left(\mathrm{~s}, 1 \mathrm{H},-\mathrm{NH}-\mathrm{SO}_{2}\right), 3.59$ (d, 2H, $\left.\mathrm{CH}_{2}\right), 3.24(\mathrm{~m}, 1 \mathrm{H}, \mathrm{HN}-\mathrm{N})$, 2.01-2.38 (s, 6H, 2 $\left.\mathrm{CH}_{3}\right)$.

$N^{4}$-(4-Fluorobenzylidene)hydrazinoacetylamino- $N^{1}$-(3,4-dimethyl-1,2-oxazol-5-yl)benzensulfonamide (4b) $\mathbf{b}_{2}$. Yield 68\%, m.p. $187-199{ }^{\circ} \mathrm{C}$; IR $\left(\mathrm{KBr}, \mathrm{cm}^{-1}\right)$ : $3452(-\mathrm{NH}), 2863\left(\mathrm{CH}_{2}-\mathrm{NH}\right), 1697(\mathrm{C}=\mathrm{O}), 1631$ (HN-CO), $1601(-\mathrm{C}=\mathrm{C}$ - aromatic ring), $1507(\mathrm{~N}=\mathrm{CH}), 1482(-\mathrm{C}=\mathrm{C}$ - oxazole ring), $1320(\mathrm{C}-\mathrm{O}), 1225$ (C-F), 1153 (-NH-SO $), 1097$ (CH aromatic ring), $962(\mathrm{~S}-\mathrm{N}), 824(\mathrm{~S}-\mathrm{C})$; ${ }^{1} \mathrm{H}-\mathrm{NMR}$ 8: 8.36 (s, 1H, $\mathrm{CO}-\mathrm{NH}), 8.21(\mathrm{~s}, 1 \mathrm{H}, \mathrm{N}=\mathrm{CH}), 7.18-7.91(\mathrm{~d}, 8 \mathrm{H}, A r-\mathrm{H}), 4.65\left(\mathrm{~s}, 1 \mathrm{H},-\mathrm{NH}-\mathrm{SO}_{2}\right), 3.62\left(\mathrm{~d}, 2 \mathrm{H}, \mathrm{CH}_{2}\right)$, $3.38(\mathrm{~m}, 1 \mathrm{H}, \mathrm{HN}-\mathrm{N}), 2.08-2.34\left(\mathrm{~s}, 6 \mathrm{H}, 2 \mathrm{CH}_{3}\right)$.

$N^{4}$-(4-Chlorobenzylidene)hydrazinoacetylamino- $N^{1}-\left(3,4-\right.$ dimethyl-1,2-oxazol-5-yl)benzensulfonamide $\quad\left(\mathbf{4} \mathbf{b}_{\mathbf{3}}\right)$. Yield 62\%, m.p. $175-178{ }^{\circ} \mathrm{C}$; IR $\left(\mathrm{KBr}, \mathrm{cm}^{-1}\right)$ : $3451(-\mathrm{NH}), 2850\left(\mathrm{CH}_{2}-\mathrm{NH}\right), 1702(\mathrm{C}=\mathrm{O}), 1622$ (HN-CO), $1586(-\mathrm{C}=\mathrm{C}$ - aromatic ring), $1540(\mathrm{~N}=\mathrm{CH}), 1483(-\mathrm{C}=\mathrm{C}$ - oxazole ring), $1312(\mathrm{C}-\mathrm{O}), 1169$ (-NH-SO ${ }_{2}$ ), 1086 (CH aromatic ring), $957(\mathrm{~S}-\mathrm{N}), 830(\mathrm{~S}-\mathrm{C}), 814(\mathrm{C}-\mathrm{Cl}) ;{ }^{1} \mathrm{H}-\mathrm{NMR}$ 8: 8.31 (s, $1 \mathrm{H}$, CO-NH), $8.16(\mathrm{~s}, 1 \mathrm{H}, \mathrm{N}=\mathrm{CH}), 7.25-7.98(\mathrm{~d}, 8 \mathrm{H}, A r-\mathrm{H}), 4.74\left(\mathrm{~s}, 1 \mathrm{H},-\mathrm{NH}-\mathrm{SO}_{2}\right), 3.64\left(\mathrm{~d}, 2 \mathrm{H}, \mathrm{CH}_{2}\right)$, $3.39(\mathrm{~m}, 1 \mathrm{H}, \mathrm{HN}-\mathrm{N}), 2.08-2.40\left(\mathrm{~s}, 6 \mathrm{H}, 2 \mathrm{CH}_{3}\right)$.

$N^{4}$-(4-Bromobenzylidene)hydrazinoacetylamino- $N^{1}$-(3,4-dimethyl-1,2-oxazol-5-yl)benzensulfonamide (4b) $\mathbf{b}_{4}$. Yield 57\%, m.p. $219{ }^{\circ} \mathrm{C}$; IR $\left(\mathrm{KBr}, \mathrm{cm}^{-1}\right)$ : $3439(-\mathrm{NH}), 2870\left(\mathrm{CH}_{2}-\mathrm{NH}\right), 1669(\mathrm{C}=\mathrm{O}), 1625$ (HN-CO), 1591 (-C=C- aromatic ring), $1522(\mathrm{~N}=\mathrm{CH}), 1481(-\mathrm{C}=\mathrm{C}-$ oxazole ring), $1315(\mathrm{C}-\mathrm{O}), 1156$ (-NH-SO ${ }_{2}$ ), 1095 (CH aromatic ring), $940(\mathrm{~S}-\mathrm{N}), 832(\mathrm{~S}-\mathrm{C}), 555(\mathrm{C}-\mathrm{Br}) ;{ }^{1} \mathrm{H}-\mathrm{NMR}$ \%: 8.32 (s, 1H, CO-NH), 8.18 (s, 1H, N=CH), 7.36-7.94 (d, 8H, Ar-H), 4.61 (s, 1H, -NH-SO $)_{2}, 3.65\left(\mathrm{~d}, 2 \mathrm{H}, \mathrm{CH}_{2}\right), 3.39$ (m, $1 \mathrm{H}, \mathrm{HN}-\mathrm{N}), 2.10-2.32\left(\mathrm{~s}, 6 \mathrm{H}, 2 \mathrm{CH}_{3}\right)$.

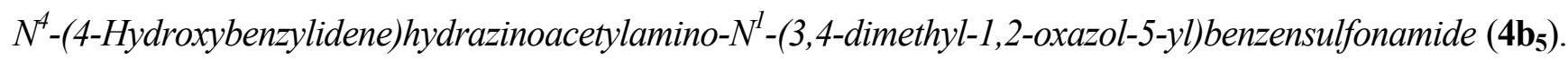
Yield 63\%, m.p. $190-192{ }^{\circ} \mathrm{C}$; IR $\left(\mathrm{KBr}, \mathrm{cm}^{-1}\right): 3495(\mathrm{C}-\mathrm{OH}), 3479(-\mathrm{NH}), 2859\left(\mathrm{CH}_{2}-\mathrm{NH}\right), 1659$ $(\mathrm{C}=\mathrm{O}), 1622(\mathrm{HN}-\mathrm{CO}), 1593(-\mathrm{C}=\mathrm{C}$ - aromatic ring), $1530(\mathrm{~N}=\mathrm{CH}), 1476(-\mathrm{C}=\mathrm{C}$ - oxazole ring), 1323 (C-O), 1150 (-NH-SO $), 1094\left(\mathrm{CH}\right.$ aromatic ring), $962(\mathrm{~S}-\mathrm{N}), 825(\mathrm{~S}-\mathrm{C}) ;{ }^{1} \mathrm{H}-\mathrm{NMR}$ 8: 8.36 (s, 1H, 
CO-NH), $8.22(\mathrm{~s}, 1 \mathrm{H}, \mathrm{N}=\mathrm{CH}), 7.31-7.84(\mathrm{~d}, 8 \mathrm{H}, A r-\mathrm{H}), 4.92$ (s, 1H, Ar-OH), 4.62 (s, 1H, -NH-SO $)_{2}$, $3.58\left(\mathrm{~d}, 2 \mathrm{H}, \mathrm{CH}_{2}\right), 3.42(\mathrm{~m}, 1 \mathrm{H}, \mathrm{HN}-\mathrm{N}), 2.08-2.32\left(\mathrm{~s}, 6 \mathrm{H}, 2 \mathrm{CH}_{3}\right)$.

$N^{4}$-(4-Nitrobenzylidene)hydrazinoacetylamino- $N^{l}$-(3,4-dimethyl-1,2-oxazol-5-yl)benzensulfonamide $\left(\mathbf{4 b}_{\mathbf{6}}\right)$. Yield 71\%, m.p. $280{ }^{\circ} \mathrm{C}$; IR (KBr, cm $\left.{ }^{-1}\right)$ : $3433(-\mathrm{NH}), 2842\left(\mathrm{CH}_{2}-\mathrm{NH}\right), 1683(\mathrm{C}=\mathrm{O}), 1629$ (HN-CO), $1593\left(-\mathrm{C}=\mathrm{C}\right.$ - aromatic ring), $1516(\mathrm{~N}=\mathrm{CH}), 1480\left(-\mathrm{C}=\mathrm{C}\right.$ - oxazole ring), $1342\left(\mathrm{C}-\mathrm{NO}_{2}\right), 1329(\mathrm{C}-\mathrm{O})$, 1161 (-NH-SO 2 ), 1106 (CH aromatic ring), 951 (S-N), $838(\mathrm{~S}-\mathrm{C}) ;{ }^{1} \mathrm{H}-\mathrm{NMR} \delta: 8.28$ (s, 1H, CO-NH), $8.21(\mathrm{~s}, 1 \mathrm{H}, \mathrm{N}=\mathrm{CH}), 7.27-7.88(\mathrm{~d}, 8 \mathrm{H}, A r-\mathrm{H}), 4.64\left(\mathrm{~s}, 1 \mathrm{H},-\mathrm{NH}-\mathrm{SO}_{2}\right), 3.56\left(\mathrm{~d}, 2 \mathrm{H}, \mathrm{CH}_{2}\right), 3.21(\mathrm{~m}, 1 \mathrm{H}$, HN-N), 2.11-2.34 (s, 6H, 2CH 3 ).

\subsubsection{Preparation of $N$-(4-aryl-3-chloro-2-oxoazetidin-1-yl)aminoacetyl Sulfonamides $\mathbf{5} \mathbf{a}_{\mathbf{1 - 6}} ; \mathbf{5} \mathbf{b}_{\mathbf{1 - 6}}$}

To a solution of $\mathrm{N}$-(arylidene)hydrazinoacetyl sulfonamides $\mathbf{4} \mathbf{a}_{1-\mathbf{6}} ; \mathbf{4} \mathbf{b}_{\mathbf{1 - 6}}(2 \mathrm{mmol})$ in anhydrous 1,4-dioxane $(50 \mathrm{~mL})$, chloracetyl chloride $(3 \mathrm{mmol})$ and triethylamine $(2 \mathrm{mmol})$ were added dropwise at $0-5{ }^{\circ} \mathrm{C}$. The mixture of reaction was stirred at room temperature for $3 \mathrm{~h}$ and the solid (triethylamine hydrochloride) was removed. The solution was heated under reflux for $5 \mathrm{~h}$ and then the solvent was evaporated under reduced pressure. The solid product was washed with water $(20 \mathrm{~mL})$, filtered off, dried and recrystallized from absolute ethanol. The progress of the reaction was monitored by silica gel coated TLC plates.

$N^{4}$-(2-Phenyl-3-chloro-4-oxoazetidin-1-yl)aminoacetylamino- $N^{l}$-(pyrimidin-2-yl)benzensulfonamide (5a $\left.\mathbf{1}\right)$. Yield 75\%, m.p. $218-220{ }^{\circ} \mathrm{C}$; IR $\left(\mathrm{KBr}, \mathrm{cm}^{-1}\right)$ : $3450(-\mathrm{NH}), 2869\left(\mathrm{CH}_{2}-\mathrm{NH}\right), 2945(=\mathrm{CH}-$ pyrimidine ring), 1745 (CO $\beta$-lactam), 1622 ( $\mathrm{HN}-\mathrm{CO}), 1591$ (-C=C- aromatic ring), 1449 (-C=C-pyrimidine ring), $1314(\mathrm{C}-\mathrm{O}), 1131$ (-NH-SO 2$), 1078$ (CH aromatic ring), $945(\mathrm{~S}-\mathrm{N}), 841(\mathrm{~S}-\mathrm{C}), 635(\mathrm{C}-\mathrm{Cl})$; ${ }^{1} \mathrm{H}-\mathrm{NMR} \delta$ : 8.49-8.72 (dm, 3H, pyrimidine ring), 8.25 (s, 1H, CO-NH), 7.21-7.96 (dm, 9H, Ar-H), $5.45\left(\mathrm{~d}, 1 \mathrm{H}, \mathrm{CH}-\mathrm{Ar}\right.$, azetidinone ring), $5.18(\mathrm{~d}, 1 \mathrm{H}, \mathrm{CH}-\mathrm{Cl}), 4.27\left(\mathrm{~s}, 1 \mathrm{H},-\mathrm{NH}-\mathrm{SO}_{2}\right), 3.75\left(\mathrm{~d}, 2 \mathrm{H}, \mathrm{CH}_{2}\right)$, $3.39(\mathrm{~m}, 1 \mathrm{H}, \mathrm{HN}-\mathrm{N}) ;{ }^{13} \mathrm{C}-\mathrm{NMR} \delta: 170.4(\mathrm{CONH}), 162.8$ (CO, $\beta$-lactam), 126.7-140.9 (12 aromatic carbons), 118.9 (1C pyrimidine ring), 154.7 (3C pyrimidine ring), $67.8(\mathrm{CH}), 62.7(\mathrm{CH}-\mathrm{Cl}), 57.7$ $\left(\mathrm{CH}_{2}\right)$; Anal. calcd for $\mathrm{C}_{21} \mathrm{H}_{19} \mathrm{O}_{4} \mathrm{~N}_{6} \mathrm{~S}$ : C 55.87, $\mathrm{H} 4.24, \mathrm{~N} 18.61$; found: C 56.02, $\mathrm{H} 4.38, \mathrm{~N} 18.48$.

$N^{4}$-[2-(4-Fluoro)phenyl-3-chloro-4-oxoazetidin-1-yl] aminoacetylamino- $N^{1}$-(pyrimidin-2-yl)benzen-sulfonamide (5a $\left.\mathbf{a}_{2}\right)$. Yield 90\%, m.p. $213-215^{\circ} \mathrm{C}$; IR $\left(\mathrm{KBr}, \mathrm{cm}^{-1}\right)$ : $3355(-\mathrm{NH}), 2831\left(\mathrm{CH}_{2}-\mathrm{NH}\right), 2943(=\mathrm{CH}-$ pyrimidine ring), 1744 (CO $\beta$-lactam), 1624 ( HN-CO), 1593 (-C=C- aromatic ring), 1508 (-C=Cpyrimidine ring), 1315 (C-O), $1230(\mathrm{C}-\mathrm{F}), 1131$ (-NH-SO 2$), 1100(\mathrm{CH}$ aromatic ring), $926(\mathrm{~S}-\mathrm{N}), 836$ (SC), $613(\mathrm{C}-\mathrm{Cl}) ;{ }^{1} \mathrm{H}-\mathrm{NMR} \quad \delta: 8.47-8.70(\mathrm{dm}, 3 \mathrm{H}$, pyrimidine ring), 8.22 (s, $1 \mathrm{H}, \mathrm{CO}-\mathrm{NH})$, 7.23-7.97 (d, 8H, $A r-\mathrm{H}), 5.35$ (d, 1H, CH- $A r$, azetidinone ring), 5.04 (d, 1H, CH-Cl), $4.37(\mathrm{~s}, 1 \mathrm{H}$, -NH-SO 2 ), 3.79 (d, 2H, CH $), 3.39(\mathrm{~m}, 1 \mathrm{H}, \mathrm{HN}-\mathrm{N})$; ${ }^{13} \mathrm{C}-\mathrm{NMR} \delta: 169.2(\mathrm{CONH}), 162.5$ (CO, $\beta$-lactam), 124.9-141.06 (12 aromatic carbons), 118.8 (1C pyrimidine ring), 151.8 (3C pyrimidine ring), 69.2 (CH), $61.04(\mathrm{CH}-\mathrm{Cl}), 55.6\left(\mathrm{CH}_{2}\right)$; Anal. calcd for $\mathrm{C}_{21} \mathrm{H}_{18} \mathrm{O}_{4} \mathrm{~N}_{6} \mathrm{SF}$ : C 53.73, H 3.86, N 17.90; found: C 53.96, H 4.06, N 18.14.

$N^{4}$-[2-(4-Chloro)phenyl-3-chloro-4-oxoazetidin-1-yl] aminoacetylamino- $N^{1}$-(pyrimidin-2-yl)benzen-

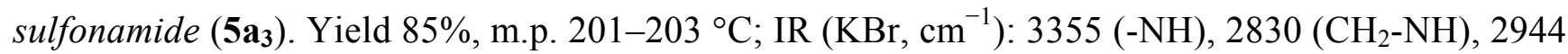


(=CH- pyrimidine ring), 1745 (CO $\beta$-lactam), 1624 (HN-CO), 1592 (-C=C- aromatic ring), 1490 (-C=C-pyrimidine ring), $1314(\mathrm{C}-\mathrm{O}), 1174\left(-\mathrm{NH}-\mathrm{SO}_{2}\right), 1088(\mathrm{CH}$ aromatic ring), $958(\mathrm{~S}-\mathrm{N}), 825(\mathrm{~S}-\mathrm{C})$, $\left.610(\mathrm{C}-\mathrm{Cl}) ;{ }^{1} \mathrm{H}-\mathrm{NMR}\right) \delta: 8.48-8.74(\mathrm{dm}, 3 \mathrm{H}$, pyrimidine ring), $8.24(\mathrm{~s}, 1 \mathrm{H}, \mathrm{CO}-\mathrm{NH}), 7.19-7.96(\mathrm{~d}, 8 \mathrm{H}$, $A r-\mathrm{H}), 5.38(\mathrm{~d}, 1 \mathrm{H}, \mathrm{CH}-A r$, azetidinone ring), 5.00 (d, 1H, CH-Cl), 4.4 (s, 1H, -NH-SO $), 3.75(\mathrm{~d}, 2 \mathrm{H}$, $\left.\mathrm{CH}_{2}\right), 3.39(\mathrm{~m}, 1 \mathrm{H}, \mathrm{HN}-\mathrm{N}) ;{ }^{13} \mathrm{C}-\mathrm{NMR} \delta: 167.6$ (CONH), 160.5 (CO, $\beta$-lactam), 126.7-142.6 (12 aromatic carbons), 120.5 (1C pyrimidine ring), 155.1 (3C pyrimidine ring), $68.7(\mathrm{CH}), 62.8$ $(\mathrm{CH}-\mathrm{Cl}), 54.4\left(\mathrm{CH}_{2}\right)$; Anal. calcd for $\mathrm{C}_{21} \mathrm{H}_{18} \mathrm{O}_{4} \mathrm{~N}_{6} \mathrm{SCl}$ : C 51.91, H 3.73, N 17.29; found: C 52.14, $\mathrm{H}$ $3.95, \mathrm{~N} 17.04$.

$N^{4}$-[2-(4-Bromo)phenyl-3-chloro-4-oxoazetidin-1-yl] aminoacetylamino- $N^{1}$-(pyrimidin-2-yl)benzensulfonamide (5-5a $)$. Yield 88\%, m.p. $206-208{ }^{\circ} \mathrm{C}$; IR (KBr, cm $\left.{ }^{-1}\right): 3445(-\mathrm{NH}), 2848\left(\mathrm{CH}_{2}-\mathrm{NH}\right), 2943$ (=CH- pyrimidine ring), 1739 (CO $\beta$-lactam), $1624(\mathrm{HN}-\mathrm{CO}), 1590(-\mathrm{C}=\mathrm{C}$ - aromatic ring), $1485(-\mathrm{C}=\mathrm{C}$ pyrimidine ring), 1315 (C-O), 1130 (-NH-SO $\left.{ }_{2}\right), 1067$ (CH aromatic ring), 961 (S-N), 840 (S-C), 630 (C-Cl), 572 (C-Br); ${ }^{1} \mathrm{H}-\mathrm{NMR}$ 8: 8.47-8.71 (dm, 3H, pyrimidine ring), 8.22 (s, 1H, CO-NH), 7.16-7.85 (d, $8 \mathrm{H}, A r-\mathrm{H}), 5.36$ (d, 1H, CH- $A r$, azetidinone ring), 5.05 (d, 1H, CH-Cl), $4.27\left(\mathrm{~s}, 1 \mathrm{H},-\mathrm{NH}-\mathrm{SO}_{2}\right), 3.79$ (d, 2H, $\mathrm{CH}_{2}$ ), 3.39 (m, 1H, HN-N); ${ }^{13} \mathrm{C}-\mathrm{NMR} \delta: 165.3$ (CONH), 160.7 (CO, $\beta$-lactam), 126.6-136.6 (12 aromatic carbons), 118.7 (1C pyrimidine ring), 156.6 (3C pyrimidine ring), $68.2(\mathrm{CH}), 62.09$ (CH-Cl), $57.5\left(\mathrm{CH}_{2}\right)$; Anal. calcd for $\mathrm{C}_{21} \mathrm{H}_{18} \mathrm{O}_{4} \mathrm{~N}_{6} \mathrm{SBr}$ : C 47.56, H 3.42, N 15.85; found: C 47.28, $\mathrm{H}$ 3.71, N 15.67 .

$N^{4}$-[2-(4-Hydroxy)phenyl-3-chloro-4-oxoazetidin-1-yl] aminoacetylamino- $N^{1}$-(pyrimidin-2-yl)benzensulfonamide (5-5a). Yield 80\%, m.p. $231-233{ }^{\circ} \mathrm{C}$; IR $\left(\mathrm{KBr}, \mathrm{cm}^{-1}\right): 3497(\mathrm{C}-\mathrm{OH}), 3355(-\mathrm{NH}), 2843$ $\left(\mathrm{CH}_{2}-\mathrm{NH}\right), 2943$ (=CH- pyrimidine ring), 1740 (CO $\beta$-lactam), $1622(\mathrm{HN}-\mathrm{CO}), 1590(-\mathrm{C}=\mathrm{C}$ - aromatic ring), 1495 (-C=C- pyrimidine ring), $1315(\mathrm{C}-\mathrm{O}), 1175$ (-NH-SO$\left.)_{2}\right), 1067$ ( $\mathrm{CH}$ aromatic ring), 961 $(\mathrm{S}-\mathrm{N}), 840(\mathrm{~S}-\mathrm{C}), 636(\mathrm{C}-\mathrm{Cl}),{ }^{1} \mathrm{H}-\mathrm{NMR} \delta: 8.54-8.75$ (dm, 3H, pyrimidine ring), $8.26(\mathrm{~s}, 1 \mathrm{H}, \mathrm{CO}-\mathrm{NH})$, 7.30-7.90 (d, 8H, $A r-\mathrm{H}), 5.34$ (d, 1H, CH- $A r$, azetidinone ring), 5.06 (d, 1H, CH-Cl), $4.95(\mathrm{~s}, 1 \mathrm{H}$, $\mathrm{Ar}-\mathrm{OH}), 4.25$ (s, 1H, -NH-SO $), 3.64$ (d, 2H, $\left.\mathrm{CH}_{2}\right), 3.40$ (m, 1H, HN-N); ${ }^{13} \mathrm{C}-\mathrm{NMR} \delta: 167.7$ (CONH), 160.3 (CO, $\beta$-lactam), 114.7-132.1 (12 aromatic carbons), 119.5 (1C pyrimidine ring), 153.4 (3C pyrimidine ring), $72.9(\mathrm{CH}), 63.3(\mathrm{CH}-\mathrm{Cl}), 54.2\left(\mathrm{CH}_{2}\right)$; Anal. calcd for $\mathrm{C}_{21} \mathrm{H}_{19} \mathrm{O}_{5} \mathrm{~N}_{6} \mathrm{~S}$ : C 53.95, H 4.10, N 17.98; found: C 54.21, H 4.32, N 18.19 .

$N^{4}$-[2-(4-Nitro)phenyl-3-chloro-4-oxoazetidin-1-yl] aminoacetylamino- $N^{1}$-(pyrimidin-2-yl)benzen-

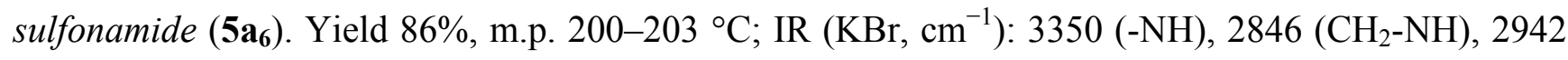
(=CH- pyrimidine ring), 1743 (CO $\beta$-lactam), $1624(\mathrm{HN}-\mathrm{CO}), 1593(-\mathrm{C}=\mathrm{C}$ - aromatic ring), $1496(-\mathrm{C}=\mathrm{C}$ pyrimidine ring), $1343\left(\mathrm{C}-\mathrm{NO}_{2}\right), 1316(\mathrm{C}-\mathrm{O}), 1131\left(-\mathrm{NH}-\mathrm{SO}_{2}\right), 1079(\mathrm{CH}$ aromatic ring), $951(\mathrm{~S}-\mathrm{N})$, 839 (S-C), 635 (C-Cl); $\left.{ }^{1} \mathrm{H}-\mathrm{NMR}\right) \delta: 8.36-8.87$ (dm, 3H, pyrimidine ring), 8.29 (s, 1H, CO-NH), 7.19-7.99 (d, 8H, $A r-\mathrm{H}), 5.33$ (d, 1H, CH- $A r$, azetidinone ring), 5.02 (d, 1H, CH-Cl), 4.24 (s, 1H, -NH-SO ${ }_{2}$ ), 3.62 (d, 2H, CH ), 3.37 (m, 1H, HN-N); ${ }^{13} \mathrm{C}-\mathrm{NMR} \delta: 168.3$ (CONH), 161.9 (CO, $\beta$-lactam), 118.5-137.2 (12 aromatic carbons), 118.6 (1C pyrimidine ring), 155.8 (3C pyrimidine ring), 76.1 $(\mathrm{CH}), 64.3(\mathrm{CH}-\mathrm{Cl}), 58.0\left(\mathrm{CH}_{2}\right)$; Anal. calcd for $\mathrm{C}_{21} \mathrm{H}_{18} \mathrm{O}_{6} \mathrm{~N}_{7} \mathrm{~S}: \mathrm{C} 50.80, \mathrm{H} 3.65, \mathrm{~N}$ 19.75; found: $\mathrm{C}$ 50.64, H 3.89, N 19.97. 
$N^{4}$-(2-Phenyl-3-chloro-4-oxoazetidin-1-yl) aminoacetylamino- $N^{l}$-(3,4-dimethyl-1,2-oxazol-5-yl)benzensulfonamide (5 $\left.\mathbf{5 b}_{\mathbf{1}}\right)$. Yield 51\%, m.p. 212-213 ${ }^{\circ} \mathrm{C}$; IR $\left(\mathrm{KBr}, \mathrm{cm}^{-1}\right): 3342(-\mathrm{NH}), 2860\left(\mathrm{CH}_{2}-\mathrm{NH}\right), 1743$ (CO $\beta$-lactam), 1624 (HN-CO), 1591 (-C=C- aromatic ring), 1495 (-C=C- oxazole ring), 1318 (C-O), 1153 (-NH-SO $), 1092$ (CH aromatic ring), 957 (S-N), 836 (S-C), $663(\mathrm{C}-\mathrm{Cl}) ;{ }^{1} \mathrm{H}-\mathrm{NMR} \delta: 8.34$ (s, 1H, CO-NH), 7.13-7.86 (dm, 9H, $A r-H), 5.37$ (d, 1H, CH- $A r$, azetidinone ring), 5.19 (d, 1H, CH-Cl), 4.57 $\left(\mathrm{s}, 1 \mathrm{H},-\mathrm{NH}-\mathrm{SO}_{2}\right), 3.51\left(\mathrm{~d}, 2 \mathrm{H}, \mathrm{CH}_{2}\right), 3.11(\mathrm{~m}, 1 \mathrm{H}, \mathrm{HN}-\mathrm{N}), 1.95-2.30\left(\mathrm{~s}, 6 \mathrm{H}, 2 \mathrm{CH}_{3}\right) ;{ }^{13} \mathrm{C}-\mathrm{NMR} \delta$ : 168.5 (CONH), 164.0 (CO, $\beta$-lactam), 151.4-155.8 (2C oxazole ring), 101.05 (1C oxazole ring), 118.8-135.3 (12 aromatic carbons), $77.2(\mathrm{CH}), 66.3(\mathrm{CH}-\mathrm{Cl}), 59.2\left(\mathrm{CH}_{2}\right), 8.48\left(2 \mathrm{CH}_{3}\right)$; Anal. calcd for $\mathrm{C}_{22} \mathrm{H}_{22} \mathrm{O}_{5} \mathrm{~N}_{5} \mathrm{~S}$ : C 56.40, H 4.73, N 14.95; found: C 56.63, H 4.94, N 15.18.

$N^{4}$-[2-(4-Fluorophenyl)-3-chloro-4-oxoazetidin-1-yl] aminoacetylamino- $N^{1}$-(3,4-dimethyl-1,2-oxazol5-yl)benzensulfonamide (5 $\left.\mathbf{5}_{\mathbf{2}}\right)$. Yield 76\%, m.p. 209-211 ${ }^{\circ} \mathrm{C}$; IR $\left(\mathrm{KBr}, \mathrm{cm}^{-1}\right): 3450(-\mathrm{NH}), 2861$ $\left(\mathrm{CH}_{2}-\mathrm{NH}\right), 1739$ (CO $\beta$-lactam), 1633 (HN-CO), 1591 (-C=C-aromatic), 1497 (-C=C- oxazole ring), 1322 (C-O), 1232 (C-F), 1153 (-NH-SO $), 1094$ (CH aromatic ring), 961 (S-N), 836 (S-C), 662 (C-Cl); ${ }^{1} \mathrm{H}-\mathrm{NMR} \delta: 8.24(\mathrm{~s}, 1 \mathrm{H}, \mathrm{CO}-\mathrm{NH}), 7.10-7.85(\mathrm{~d}, 8 \mathrm{H}, A r-\mathrm{H}), 5.38$ (d, $1 \mathrm{H}, \mathrm{CH}-A r$, azetidinone ring), 5.23 (d, 1H, CH-Cl), 4.57 (s, 1H, -NH-SO $), 3.57$ (d, 2H, $\left.\mathrm{CH}_{2}\right), 3.41(\mathrm{~m}, 1 \mathrm{H}, \mathrm{HN}-\mathrm{N}), 2.02-2.27(\mathrm{~s}, 6 \mathrm{H}$, $\left.2 \mathrm{CH}_{3}\right) ;{ }^{13} \mathrm{C}-\mathrm{NMR} \delta$ : $169.6(\mathrm{CONH}), 160.3$ (CO, $\beta$-lactam), 118.6-139.9 (12 aromatic carbons), 99.56 (1C oxazole ring), 151.8-153.3 (2C oxazole ring), $75.9(\mathrm{CH}), 64.2(\mathrm{CH}-\mathrm{Cl}), 56.2\left(\mathrm{CH}_{2}\right), 10.84\left(2 \mathrm{CH}_{3}\right)$; Anal. calcd for $\mathrm{C}_{22} \mathrm{H}_{21} \mathrm{O}_{5} \mathrm{~N}_{5} \mathrm{SF}$ : C 54.31, H 4.35, N 14.40; found: C 54.09, H 4.58, N 14.68.

$N^{4}$-[2-(4-Chlorophenyl)-3-chloro-4-oxoazetidin-1-yl] aminoacetylamino- $N^{1}$-(3,4-dimethyl-1,2-oxazol5-yl)benzensulfonamide $\left(\mathbf{5 b}_{\mathbf{3}}\right)$. Yield 72\%, m.p. 211-213 ${ }^{\circ} \mathrm{C}$; IR $\left(\mathrm{KBr}, \mathrm{cm}^{-1}\right): 3361(-\mathrm{NH}), 2869$ $\left(\mathrm{CH}_{2}-\mathrm{NH}\right), 1750$ (CO $\beta$-lactam), 1620 (HN-CO), 1591 (-C=C-aromatic), 1495 (-C=C oxazole ring), 1318 (C-O), 1153 (-NH-SO $), 1092$ (CH aromatic ring), 957 (S-N), 836 (S-C), 663 (C-Cl); ${ }^{1} \mathrm{H}-\mathrm{NMR} \delta$ : $8.23(\mathrm{~s}, 1 \mathrm{H}, \mathrm{CO}-\mathrm{NH}), 7.22-7.92(\mathrm{~d}, 8 \mathrm{H}, A r-\mathrm{H}), 5.35$ (d, 1H, CH-Ar, azetidinone ring), $5.20(\mathrm{~d}, 1 \mathrm{H}$, $\mathrm{CH}-\mathrm{Cl}), 4.68$ (s, 1H, -NH-SO $)_{2}, 3.52$ (d, 2H, $\left.\mathrm{CH}_{2}\right), 3.33(\mathrm{~m}, 1 \mathrm{H}, \mathrm{HN}-\mathrm{N}), 2.05-2.36\left(\mathrm{~s}, 6 \mathrm{H}, 2 \mathrm{CH}_{3}\right)$; ${ }^{13} \mathrm{C}-\mathrm{NMR}$ (DMSO) $\delta$ in ppm: $170.3(\mathrm{CONH}), 163.6$ (CO, $\beta$-lactam), 118.8-132.7 (12 aromatic carbons), 100.97 (1C oxazole ring), 150.4-152.7 (2C oxazole ring), $77.4(\mathrm{CH}), 66.3(\mathrm{CH}-\mathrm{Cl}), 53.1$ $\left(\mathrm{CH}_{2}\right), 8.50\left(2 \mathrm{CH}_{3}\right)$; Anal. calcd for $\mathrm{C}_{22} \mathrm{H}_{21} \mathrm{O}_{5} \mathrm{~N}_{5} \mathrm{SCl}$ : C 52.54, $\mathrm{H} \mathrm{4.21,} \mathrm{N} \mathrm{13.92;} \mathrm{found:} \mathrm{C} 52.38, \mathrm{H}$ 4.08, N 13.71 .

$N^{4}$-[2-(4-Bromophenyl)-3-chloro-4-oxoazetidin-1-yl] aminoacetylamino- $N^{1}$-(3,4-dimethyl-1,2-oxazol5-yl)benzensulfonamide (5 $\left.\mathbf{5 b}_{\mathbf{4}}\right)$. Yield 80\%, m.p. 212-214 ${ }^{\circ} \mathrm{C}$; IR $\left(\mathrm{KBr}, \mathrm{cm}^{-1}\right): 3386(-\mathrm{NH}), 2866$ $\left(\mathrm{CH}_{2}-\mathrm{NH}\right), 1748$ (CO $\beta$-lactam), 1626 (HN-CO), 1589 (-C=C- aromatic ring), 1483 (-C=C- oxazole ring), $1315(\mathrm{C}-\mathrm{O}), 1158$ (-NH-SO$), 1095(\mathrm{CH}$ aromatic ring), $960(\mathrm{~S}-\mathrm{N}), 859(\mathrm{~S}-\mathrm{C}), 660(\mathrm{C}-\mathrm{Cl}), 556$ (C-Br); ${ }^{1} \mathrm{H}-\mathrm{NMR} \delta: 8.21$ (s, 1H, CO-NH), 7.34-7.98 (d, 8H, $\left.A r-\mathrm{H}\right), 5.33$ (d, 1H, CH-Ar, azetidinone ring), 5.19 (d, 1H, CH-Cl), $4.56\left(\mathrm{~s}, 1 \mathrm{H},-\mathrm{NH}-\mathrm{SO}_{2}\right), 3.60$ (d, 2H, $\left.\mathrm{CH}_{2}\right), 3.36(\mathrm{~m}, 1 \mathrm{H}, \mathrm{HN}-\mathrm{N}), 2.08-2.28$ (s, $\left.6 \mathrm{H}, 2 \mathrm{CH}_{3}\right) ;{ }^{13} \mathrm{C}-\mathrm{NMR} \delta: 171.3(\mathrm{CONH}), 160.7$ (CO, $\beta$-lactam), 128.8-142.7 (12 aromatic carbons), 102.37 (1C oxazole ring), 151.7-153.5 (2C oxazole ring), $78.8(\mathrm{CH}), 67.0(\mathrm{CH}-\mathrm{Cl}), 55.0\left(\mathrm{CH}_{2}\right), 8.50$ $\left(2 \mathrm{CH}_{3}\right)$; Anal. calcd for $\mathrm{C}_{22} \mathrm{H}_{21} \mathrm{O}_{5} \mathrm{~N}_{5} \mathrm{SBr}$ : C 48.27, H 3.87, N 12.79; found: C 48.54, H 3.94, N 12.97.

$N^{4}$-[2-(4-Hydroxyphenyl)-3-chloro-4-oxoazetidin-1-yl] aminoacetylamino- $N^{1}$-(3,4-dimethyl-1,2-oxazol-

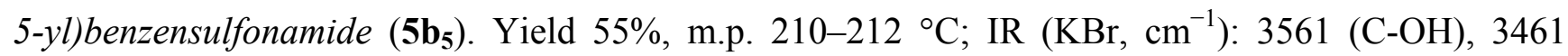


(-NH), $2855\left(\mathrm{CH}_{2}-\mathrm{NH}\right), 1746$ (CO $\beta$-lactam), 1621 (HN-CO), 1590 (-C=C- aromatic), 1480 (-C=Coxazole ring), 1308 (C-O), 1151 (-NH-SO 2$), 1093$ (CH aromatic ring), 989 (S-N), 835 (S-C), 659 (C-Cl); ${ }^{1} \mathrm{H}-\mathrm{NMR} \delta$ in ppm: $8.31(\mathrm{~s}, 1 \mathrm{H}, \mathrm{CO}-\mathrm{NH}), 7.28-7.81(\mathrm{~d}, 8 \mathrm{H}, A r-\mathrm{H}), 5.32(\mathrm{~d}, 1 \mathrm{H}, \mathrm{CH}-A r$, azetidinone ring), $5.10(\mathrm{~d}, 1 \mathrm{H} \mathrm{CH}-\mathrm{Cl}), 4.84(\mathrm{~s}, 1 \mathrm{H}, \mathrm{Ar}-\mathrm{OH}), 4.57\left(\mathrm{~s}, 1 \mathrm{H},-\mathrm{NH}-\mathrm{SO}_{2}\right), 3.55\left(\mathrm{~d}, 2 \mathrm{H}, \mathrm{CH}_{2}\right), 3.39(\mathrm{~m}$, 1H, HN-N), 2.04-2.28 (s, 6H, 2CH3); ${ }^{13} \mathrm{C}-\mathrm{NMR} \delta: 175.2$ (CONH), 168.2 (CO, $\beta$-lactam), 115.8-126.8 (12 aromatic carbons), 101.79 (1C oxazole ring), 148.9-150.5 (2C oxazole ring), $77.3(\mathrm{CH}), 64.8$ $(\mathrm{CH}-\mathrm{Cl}), 55.2\left(\mathrm{CH}_{2}\right), 8.51\left(2 \mathrm{CH}_{3}\right)$; Anal. calcd for $\mathrm{C}_{22} \mathrm{H}_{22} \mathrm{O}_{6} \mathrm{~N}_{5} \mathrm{~S}$ : C 54.54, $\mathrm{H} 4.58, \mathrm{~N} 14.45$; found: $\mathrm{C}$ $54.73, \mathrm{H} 4.63, \mathrm{~N} 14.71$.

$N^{4}$-[2-(4-Nitrophenyl)-3-chloro-4-oxoazetidin-1-yl] aminoacetylamino- $N^{1}-(3,4-d i m e t h y l-1,2-o x a z o l-5$ -

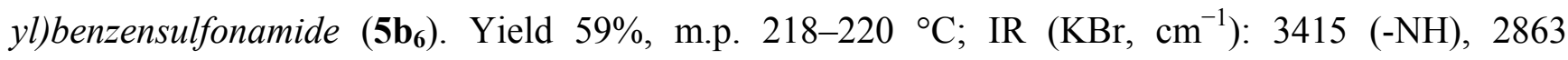
( $\left.\mathrm{CH}_{2}-\mathrm{NH}\right), 1752$ (CO $\beta$-lactam), 1624 (HN-CO), 1591 (-C=C- aromatic ring), 1490 (-C=C- oxazole ring), 1337 (C-O), $1329\left({\left.\mathrm{C}-\mathrm{NO}_{2}\right)}\right) 154\left(-\mathrm{NH}-\mathrm{SO}_{2}\right), 1092(\mathrm{CH}$ aromatic ring), 987 (S-N), 836 (S-C), $662(\mathrm{C}-\mathrm{Cl}) ;{ }^{1} \mathrm{H}-\mathrm{NMR} \delta: 8.18(\mathrm{~s}, 1 \mathrm{H}, \mathrm{CO}-\mathrm{NH}), 7.19-7.86(\mathrm{~d}, 8 \mathrm{H}, A r-\mathrm{H}), 5.36(\mathrm{~d}, 1 \mathrm{H}, \mathrm{CH}-A r$, azetidinone ring), $5.20(\mathrm{~d}, 1 \mathrm{H}, \mathrm{CH}-\mathrm{Cl}), 4.55\left(\mathrm{~s}, 1 \mathrm{H},-\mathrm{NH}-\mathrm{SO}_{2}\right), 3.49\left(\mathrm{~d}, 2 \mathrm{H}, \mathrm{CH}_{2}\right), 3.11(\mathrm{~m}, 1 \mathrm{H}$, $\mathrm{HN}-\mathrm{N}) ; 2.09-2.27$ (s, 6H, 2CH 3 ); ${ }^{13} \mathrm{C}-\mathrm{NMR} \delta: 170.7$ (CONH), 165.1 (CO, $\beta$-lactam), 118.7-130.6 (12 aromatic carbons), 101.20 (1C oxazole ring), 148.8-150.4 (2C oxazole ring), $79.1(\mathrm{CH}), 65.4(\mathrm{CH}-\mathrm{Cl})$, $60.7\left(\mathrm{CH}_{2}\right), 9.54\left(2 \mathrm{CH}_{3}\right)$; Anal. calcd for $\mathrm{C}_{22} \mathrm{H}_{21} \mathrm{O}_{7} \mathrm{~N}_{6} \mathrm{~S}: \mathrm{C} 51.46, \mathrm{H} 4.12, \mathrm{~N} \mathrm{16.37}$; found: $\mathrm{C} 51.29, \mathrm{H}$ 4.36, N 16.09 .

\subsection{Biological Evaluation}

\subsubsection{Antibacterial Assay}

In order to evaluate the antibacterial activity of the synthesized compounds $\mathbf{4} \mathbf{a}_{1-6}, \mathbf{4} \mathbf{b}_{1-\mathbf{6}}, \mathbf{5} \mathbf{a}_{1-6}$ and $\mathbf{5 b}_{1-6}$, a panel of three Gram positive (Staphyloccoccus aureus ATCC 6583, Staphyloccoccus epidermidis ATCC 12228, Enterococcus faecalis ATCC 25912) and six Gram negative (Klebsiella pneumoniae CIP 53153, Proteus vulgaris CIP 104989, Citrobacter freundii CIP 5732, Enterobacter cloacae CIP 103475, Escherichia coli ATCC 25922, Pseudomonas aeruginosa CIP 82118) bacterial strains were used. Minimum inhibitory concentrations (MICs) were assessed according to the guidelines of EUCAST Def. 3.1 [24]. Briefly, stock solutions were prepared by solving the substances mentioned above $(200 \mathrm{mg}$ ) in dimethyl sulfoxide (DMSO, $19.5 \mathrm{~mL}$ ). Using these solutions, series of two-fold dilutions were subsequently obtained. In a $9 \mathrm{~cm}$ diameter Petri dish, one milliliter of each dilution was mixed thoroughly with Mueller-Hinton agar $(19 \mathrm{~mL})$, sterilized by autoclaving and cooled to $50{ }^{\circ} \mathrm{C}$. After this, the concentrations of the substances inside the medium were 512, 256, 128, 64, 32, 16, 8, 4, 2 , and $1 \mu \mathrm{g} / \mathrm{mL}$ respectively. A blank plate (control of growth) was also prepared by mixing DMSO $(1 \mathrm{~mL})$ with molten agar $(19 \mathrm{~mL})$. From each bacterial strain, a 0.5 McFarland suspension was prepared in $0.85 \%$ saline solution and after that, the inoculum was standardized in order to assure $10^{4}$ colony-forming units $(\mathrm{CFU})$ per spot $(5 \mu \mathrm{L})$. All inoculated plates were incubated for $18 \mathrm{~h}$ at $36{ }^{\circ} \mathrm{C}$. The MIC was interpreted as the lowest concentration of the substance that completely inhibits the growth of bacteria in the spot area. Each determination was performed in triplicate in order to accurately confirm the MIC values. 


\subsubsection{Antioxidant Assays}

The antioxidant activity was estimated using in vitro tests: ferric reducing power, total antioxidant capacity and radical scavenging ability.

\subsubsection{Ferric Reducing Power}

The ferric reducing power of the compounds was quantified by the method described by [25] with slight modifications. The sample solution ( $1 \mathrm{~mL}, 5 \mathrm{mg} / \mathrm{mL}$ in DMSO) was mixed with sodium phosphate buffer $(1 \mathrm{~mL}, 0.2 \mathrm{M}, \mathrm{pH} 6.6)$ and potassium ferricyanide $(1 \mathrm{~mL}, 1 \% \mathrm{w} / \mathrm{v})$ in a test tube. The reaction mixture was incubated at $50{ }^{\circ} \mathrm{C}$ for $20 \mathrm{~min}$ in a water bath and then the reaction was stopped by adding trichloroacetic acid $(1 \mathrm{~mL}, 10 \% \mathrm{w} / \mathrm{v})$. After centrifugation of the mixture at $4500 \mathrm{rpm}$ for $15 \mathrm{~min}$, the upper layer of the solution $(1 \mathrm{~mL})$ was collected and diluted further by adding deionised water $(1 \mathrm{~mL})$ and ferric chloride $(0.2 \mathrm{~mL}, 0.1 \% \mathrm{w} / \mathrm{v})$. After $5 \mathrm{~min}$ of incubation, the absorbance was measured at $700 \mathrm{~nm}$ against a blank (the mixture of DMSO with the reagents). A higher absorbance indicates a higher reducing power. For each sample it was calculated the effective concentration $\left(\mathrm{EC}_{50}\right)$ and the reducing power was expressed in reference with ascorbic acid (AA) in the same concentration.

\subsubsection{Total Antioxidant Activity}

The antioxidant activity of tested compounds was evaluated using the phosphomolybdenum method according to the procedure of [26] with minor modifications. The method is based on the reduction of $\mathrm{Mo}(\mathrm{VI})$ to $\mathrm{Mo}(\mathrm{V})$ by the tested compounds followed by the formation of a green phosphate/Mo(V) complex at acid $\mathrm{pH}$. An aliquot of sample solution $(50 \mu \mathrm{L}, 5 \mathrm{mg} / \mathrm{mL}$ in DMSO) is mixed with the reagent solution $(2 \mathrm{~mL}, 0.6 \mathrm{M}$ sulphuric acid, $28 \mathrm{mM}$ sodium phosphate and $4 \mathrm{mM}$ ammonium molybdate). The samples were incubated at $95{ }^{\circ} \mathrm{C}$ for $90 \mathrm{~min}$ and then were cooled to room temperature. The absorbance was measured at $695 \mathrm{~nm}$ against a blank (DMSO mixed with reagent solution). For each sample the effective concentration $\left(\mathrm{EC}_{50}\right)$ was calculated and the antioxidant activity was expressed in reference with ascorbic acid (AA) in the same concentration.

\subsubsection{DPPH Radical Scavenging Assay}

The radical scavenging activity of the tested compounds towards the radical 1,1-diphenyl-2picrylhydrazyl (DPPH) was measured as described by [27] with slight modifications. The sample solution $(50 \mu \mathrm{L}, 20 \mathrm{mg} / \mathrm{mL}$ in DMSO) was mixed thoroughly with a solution of DPPH in methanol (2.95 mL, $0.1 \mathrm{mM})$. The sample was left for $30 \mathrm{~min}$ at room temperature, in the dark, and after that the absorbance was measured at $517 \mathrm{~nm}\left(\mathrm{~A}_{\mathrm{s}}\right)$. A methanol solution of DPPH was used as control sample $\left(\mathrm{A}_{\mathrm{c}}\right)$. The ability to scavenge the DPPH radical was calculated using the following formula:

$$
\% \text { Inhibition }=100 \times(\mathrm{Ac}-\mathrm{As}) / \mathrm{Ac}
$$

and it was expressed in reference with the radical scavenging activity of ascorbic acid (AA) in the same concentration. 


\subsubsection{Statistical Analysis}

All assays (antimicrobial and antioxidant) were carried out in triplicate. Data were analysed by an analysis of variance (ANOVA) $(p<0.05)$ and were expressed as means \pm SD. The total antioxidant antivity $\left(\mathrm{EC}_{50}\right.$ values) were calculated by linear interpolation between values above and below $50 \%$ activity.

\section{Conclusions}

In this study new $N$-(arylidene)hydrazinoacetyl and new 2-azetidionone derivatives have been designed and synthesized starting from sulfadiazine and sulfizoxazole. The structures of all new compounds were proved using spectral methods. The compounds were evaluated for their antimicrobial and antioxidant activity. Although their antimicrobial potential was reduced, they shown excellent antioxidant properties; for some of them the potential is comparable with the antioxidant activity of ascorbic acid. These results support the antioxidant potential of the synthesized compounds and their applications in several disease mediated by reactive oxygen species (ROS) including the healing of the wounds.

\section{Acknowledgments}

This work was supported in part by the project "Doctoral Scholarships for increasing competitiveness in the medical and pharmaceutical field" (POSDRU/88/1.5/S/58965) and the grant of the Romanian National Authority for Scientific Research, CNCS - UEFISCDI, project number PN-IIID-PCE-2011-3-0906 (Contract No. 274/31.10.2011). The authors thank Polymer Research Group, Department of Organic Chemistry, University of Ghent, Belgium for their help to perform NMR analyses.

\section{Conflict of Interest}

The authors declared no conflict of interest.

\section{References}

1. Chavan, A.A.; Pai, N.R. Synthesis and biological activity of N-substituted-3-chloro-2azetidinones. Molecules 2007, 12, 2467-2477.

2. Ishwar, B.K.; Mishra, S.K.; Jainey, P.J.; Shastry, C.S. Antimicrobial studies of synthesized azetidinone derivatives from sulfamethoxazole moiety. J. Chem. Pharm. Res. 2011, 3, 114-118.

3. Jarrahpour, A.; Zarei, M. Synthesis of novel $N$-sulfonyl monocyclic $\beta$-lactams as potential antibacterial agents. Molecules 2006, 11, 49-58.

4. Cerić, H.; Šindler-Kulyk, M.; Kovačević, M.; Perić, M.; Živković, A. Azetidinone-isothiazolidinones: Stereoselective synthesis and antibacterial evaluation of new monocyclic beta-lactams. Bioorg. Med. Chem. 2010, 18, 3053-3058.

5. Sharma, R.; Samadhiya, P.; Srivastava, S.D.; Srivastava, S.K. Synthesis and pharmaceutical importance of 2-azetidinone derivatives of phenothiazine. J. Chem. Sci. 2012, 124, 633-637. 
6. Samadhiya, P.; Sharma, R.; Srivastava, S.K.; Srivastava, S.D. Synthesis of 2-azetidinone derivatives of 6-nitro-1H-indazole and their biological importance. Quím. Nova 2012, 35, 914-919.

7. Kumar, A.; Rajput, C.S.; Bhati, S.K. Synthesis of 3-[4'-(p-chlorophenyl)-thiazol-2'-yl]-2[(substituted azetidinone/thiazolidinone)-aminomethyl]-6-bromoquinazolin-4-ones as anti-inflammatory agent. Bioorg. Med. Chem. 2007, 15, 3089-3096.

8. Smith, E.M.; Sorota, S.; Kim, H.M.; McKittrick, B.A.; Nechuta, T.L.; Bennett, C.; Knutson, C.; Burnett, D.A.; Kieselgof, J.; Tan, Z.; et al. T-type calcium channel blockers: Spiro-piperidine azetidines and azetidinones-optimization, Design and synthesis. Bioorg. Med. Chem. Lett. 2010, 20, 4602-4606.

9. Aoyama, Y.; Uenaka, M.; Kii, M.; Tanaka, M.; Konoike, T.; Hayasaki-Kajiwara, Y.; Naya, N.; Nakajima, M. Design, Synthesis and pharmacological evaluation of 3-benzylazetidine-2-onebased human chymase inhibitors. Bioorg. Med. Chem. 2001, 9, 3065-3075.

10. O’Boyle, N.M.; Greene, L.M.; Bergin, O.; Fichet, J.-B.; McCabe, T.; Lloyd, D.G.; Zisterer, D.M.; Meegan, M.J. Synthesis, Evaluation and structural studies of antiproliferative tubulin-targeting azetidin-2-ones. Bioorg. Med. Chem. 2011, 19, 2306-2325.

11. Galletti, P.; Quintavalla, A.; Ventrici, C.; Giannini, G.; Cabri, W.; Penco, S.; Gallo, G.; Vincenti, S.; Giacomini, D. Azetidinones as zinc-binding groups to design selective HDAC8 inhibitors. Chem. Med. Chem. 2009, 4, 1991-2001.

12. Tripodi, F.; Pagliarin, R.; Fumagalli, G.; Bigi, A.; Fusi, P.; Orsini, F.; Frattini, M.; Coccetti, P. Synthesis and biological evaluation of 1,4-diaryl-2-azetidinones as specific anticancer agents: activation of adenosine monophosphate activated protein kinase and induction of apoptosis. J. Med. Chem. 2012, 55, 2112-2124.

13. Mehta, P.D.; Sengar, N.P.; Pathak, A.K. 2-Azetidinone-a new profile of various pharmacological activities. Eur. J. Med. Chem. 2010, 45, 5541-5560.

14. Gavernet, L.; Barrios, I.A.; Cravero, M.S.; Bruno-Blanch, L.E. Design, Synthesis, and anticonvulsant activity of some sulfamides. Bioorg. Med. Chem. 2007, 15, 5604-5614.

15. Chohan, Z.H. Metal-based antibacterial and antifungal sulfonamides: Synthesis, Characterization, and biological properties. Transit. Metal Chem. 2009, 34, 153-161.

16. Sasidharan, S.; Logeswaran S.; Latha, L.Y. Wound healing activity of Elaeis Guineensis leaf extract ointment. Int. J. Mol. Sci. 2012, 13, 336-347.

17. Schönfelder, U.; Abel, M.; Wiegand, C.; Klemm, D.; Elsner, P.; Hipler, U.-C. Influence of selected wound dressings on PMN elastase in chronic wound fluid and their antioxidative potential in vitro. Biomaterials 2005, 26, 6664-6673.

18. Melo-Silveira, R.F.; Fidelis, G.P.; Costa, M.S.; Telles, C.B.; Dantas-Santos, N.; de Oliveira Elias, S.; Ribeiro, V.B.; Barth, A.L.; Macedo, A.J.; Leite, E.L.; et al. In vitro antioxidant, Anticoagulant and antimicrobial activity and in inhibition of cancer cell proliferation by xylan extracted from Corn Cobs. Int. J. Mol. Sci. 2012, 13, 409-426.

19. Roy, P.; Amdekar, S.; Kumar, A.; Singh, R.; Sharma, P.; Singh, V. In vivo antioxidative property, antimicrobial and wound healing activity of flower extracts of Pyrostegia venusta (Ker Gawl) Miers. J. Ethnopharmacol. 2012, 140, 186-192.

20. Ghaisas, M.M.; Kshirsagar, S.B.; Sahane, R.S. Evaluation of wound healing activity of ferulic acid in diabetic rats. Int. Wound. J. 2012, doi:10.1111/j.1742-481X.2012.01119.x. 
21. Chigurupati, S.; Mughal, M.R.; Chan, S.L.; Arumugam, T.V.; Baharani, A.; Tang, S.C.; Yu, Q.S.; Holloway, H.W.; Wheeler, R.; Poosala, S.; et al. A synthetic uric acid analog accelerates cutaneous wound healing in mice. PLoS One 2010, 5, e10044.

22. Naik, H.R.; Naik, H.S.; Naik, T.R.; Naika, H.R.; Gouthamchandra, K.; Mahmood, R.; Ahamed, B.M. Synthesis of novel benzo[h]quinolines: Wound healing, Antibacterial, DNA binding and in vitro antioxidant activity. Eur. J. Med. Chem. 2009, 44, 981-989.

23. Parasca, O.M.; Lupascu, F.; Vasile, C.; Mares, M.; Nastasa, V.; Profire, L. New hydrazynes with sulfonamidic structure: Synthesis, Characterization and biological activity. Rev. Med. Chir. 2013, in press.

24. European Committee for Antimicrobial Susceptibility Testing (EUCAST) of the European Society for Clinical Microbiology and Infectious Diseases (ESCMID). EUCAST Definitive Document E. Def 3.1.: Determination of minimum inhibitory concentrations (MICs) of antibacterial agents by agar dilution. Clin. Microbiol. Infect. 2000, 6, 509-515.

25. Cacic, M.; Molnar, M.; Sarkanj, B.; Has-Schon, E.; Rajkovic, V. Synthessis and antioxidant activity of some new coumarinyl-1,3-Thiazolidine-4-ones. Molecules 2010, 15, 6795-6809.

26. Cervellati, R.; Galletti, P.; Greco, E.; Cocuzza, C.E.; Musumeci, R.; Bardini, L.; Paolucci, F.; Pori, M.; Soldati, R.; Giacomini, D. Monocyclic $\beta$-lactams as antibacterial agents: Facing antioxidant activity of N-methylthio-azetidinones. Eur. J. Med. Chem. 2012, 60, 340-349.

27. Osorio, M.; Aravena, J.; Vergara A.; Taborga, L.; Baeza, E.; Catalán, K.; González, C.; Carvajal, M.; Carrasco. H.; Espinoza, L. Synthesis and DPPH radical scavenging activity of prenylated phenol derivatives. Molecules 2012, 17, 556-570.

Sample Availability: Samples of the compounds $\mathbf{4} \mathbf{a}_{1-6}, \mathbf{4} \mathbf{b}_{\mathbf{1 - 6}}, \mathbf{5} \mathbf{a}_{1-\mathbf{6}}, \mathbf{5} \mathbf{b}_{\mathbf{1 - 6}}$ are available from the authors.

(C) 2013 by the authors; licensee MDPI, Basel, Switzerland. This article is an open access article distributed under the terms and conditions of the Creative Commons Attribution license (http://creativecommons.org/licenses/by/3.0/). 